\title{
Resistance and resilience to Alzheimer's disease pathology are associated with reduced cortical pTau and absence of limbic-predominant age-related TDP-43 encephalopathy in a community-based cohort
}

Caitlin S. Latimer $^{1^{*}}$ D, Bridget T. Burke², Nicole F. Liachko ${ }^{3,4}$, Heather N. Currey ${ }^{3}$, Mitchell D. Kilgore ${ }^{1}$, Laura E. Gibbons ${ }^{5}$, Jonathan Henriksen ${ }^{1}$, Martin Darvas ${ }^{1}$, Kimiko Domoto-Reilly ${ }^{6}$, Suman Jayadev ${ }^{6}$, Tom J. Grabowski, ${ }^{6,7}$ Paul K. Crane ${ }^{5}$, Eric B. Larson², Brian C. Kraemer ${ }^{3,4,8}$, Thomas D. Bird ${ }^{3,6,9}$ and C. Dirk Keene ${ }^{1}$

\begin{abstract}
Alzheimer's disease neuropathologic change (ADNC) is defined by progressive accumulation of $\beta$-amyloid plaques and hyperphosphorylated tau (pTau) neurofibrillary tangles across diverse regions of brain. Non-demented individuals who reach advanced age without significant $A D N C$ are considered to be resistant to $A D$, while those burdened with ADNC are considered to be resilient. Understanding mechanisms underlying ADNC resistance and resilience may provide important clues to treating and/or preventing AD associated dementia. ADNC criteria for resistance and resilience are not well-defined, so we developed stringent pathologic cutoffs for non-demented subjects to eliminate cases of borderline pathology. We identified 14 resistant (85+ years old, non-demented, Braak stage $\leq I I I$, CERAD absent) and 7 resilient (non-demented, Braak stage VI, CERAD frequent) individuals out of 684 autopsies from the Adult Changes in Thought study, a long-standing community-based cohort. We matched each resistant or resilient subject to a subject with dementia and severe ADNC (Braak stage VI, CERAD frequent) by age, sex, year of death, and post-mortem interval. We expanded the neuropathologic evaluation to include quantitative approaches to assess neuropathology and found that resilient participants had lower neocortical pTau burden despite fulfilling criteria for Braak stage VI. Moreover, limbic-predominant age-related TDP-43 encephalopathy neuropathologic change (LATE-NC) was robustly associated with clinical dementia and was more prevalent in cases with high pTau burden, supporting the notion that resilience to ADNC may depend, in part, on resistance to pTDP43 pathology. To probe for interactions between tau and TDP-43, we developed a C. elegans model of combined human (h) Tau and TDP-43 proteotoxicity, which exhibited a severe degenerative phenotype most compatible with a synergistic, rather than simply additive, interaction between hTau and hTDP-43 neurodegeneration. Pathways that underlie this synergy may present novel therapeutic targets for the prevention and treatment of AD.
\end{abstract}

Keywords: Resistance, Resilience, Alzheimer's disease neuropathologic change, TDP-43, Hyperphosphorylated tau, Dementia, C. elegans

\footnotetext{
*Correspondence: caitlinl@uw.edu

'Division of Neuropathology, Department of Pathology, University of

Washington, Seattle, WA 98104, USA

Full list of author information is available at the end of the article
}

C The Author(s). 2019 Open Access This article is distributed under the terms of the Creative Commons Attribution 4.0 International License (http://creativecommons.org/licenses/by/4.0/), which permits unrestricted use, distribution, and reproduction in any medium, provided you give appropriate credit to the original author(s) and the source, provide a link to the Creative Commons license, and indicate if changes were made. The Creative Commons Public Domain Dedication waiver (http://creativecommons.org/publicdomain/zero/1.0/) applies to the data made available in this article, unless otherwise stated. 


\section{Introduction}

Alzheimer's disease neuropathologic change (ADNC) is defined by the presence of extracellular amyloid (A) $\beta$ plaques with varying levels of intracellular neurofibrillary tangles of hyperphosphorylated tau (pTau) present in well-characterized, stereotypical patterns throughout the brain. In general, the more extensively the brain is involved by $A \beta$ and pTau pathology, the greater the likelihood an individual was cognitively impaired or demented as a result of the clinical manifestations of Alzheimer's disease (AD). These clinical-neuropathologic correlations have been described in diverse autopsy cohort studies and are summarized in the 2012 NIA-AA guidelines for the neuropathological evaluation of $\mathrm{AD}$ [55]. While the vast majority of individuals over the age of 85 at death have some degree of AD neuropathology, those who have at least an intermediate degree of ADNC as defined by the NIA-AA guidelines are considered to have sufficient pathologic burden to explain dementia prior to death. However, there are some individuals with no clinical history of dementia who, at autopsy, are found to have intermediate or even high ADNC; although there is not a widely accepted set of criteria, these individuals may be considered to be resilient to the pathologic changes of $\mathrm{AD}[5,8,23,62,82]$. Conversely, some people, despite advanced age, do not develop ADNC and can be described as resistant to developing $\mathrm{AD}$ [48]. Although under these definitions, resilient and resistant individuals are relatively few in number in autopsy cohorts and represent the extremes of the population, understanding mechanisms that underlie their resilience or resistance to $\mathrm{ADNC}$, which are likely to be distinct, may provide important clues to develop preventive and therapeutic strategies for $A D$.

Community-based cohort studies have shown that the neuropathologic changes of AD rarely exist in isolation; rather, the majority of elderly individuals are found to have multiple comorbid pathologies at autopsy, most commonly Lewy body disease (LBD), vascular brain injury (VBI) including microvascular brain injury ( $\mu \mathrm{VBI})$, and pTDP-43 pathology, with or without hippocampal sclerosis $[14,19,35,41,43,63,78,85,98,99,101]$. The latter has recently been described as limbic-predominant age-related TDP-43 encephalopathy neuropathological change (LATE-NC) defined by a stereotypically distributed TDP-43 proteinopathy in older adults [66]. An active area of research is to determine if or how these diverse pathologies interact, possibly even synergize, to accelerate neurotoxicity and neurodegeneration and eventually functional (cognitive) decline. Individuals with multiple co-morbid pathologies are more likely to experience cognitive symptoms [56, 91]; conversely, those who reach advanced age without cognitive impairment harbor fewer co-existing pathologies on average compared to those with dementia [48, 92]. Therefore, what appears to be cognitive resilience to ADNC may, in part, be due to resistance to developing comorbid neuropathologies. Mechanisms underlying these processes are unknown, but some studies have identified relationships of resilience and resistance to ADNC with systemic exposures and disorders including smoking, cardiovascular disease, diabetes, and medication history $[50,56]$, as well as lifetime experiences [15, 24, 28, 44, 72, 75, 89], such as educational level, occupation, intelligence, degree of involvement in socially, physically, and cognitively stimulating activities, socioeconomic status, and early life environment.

The concepts of resistance and resilience can be defined and interpreted in many ways, but for the purposes of this study we refer specifically to resistance to the development of and resilience to existing ADNC and associated cognitive impairment. Even with this focused definition of resistance and resilience, we recognize that there are still challenges in applying these definitions in research cohorts given imprecise, qualitative neuropathological criteria for AD regarding pathologic burden and the potential to overclassify resilience and resistance based on less stringent criteria for both pathologic changes and clinical characterization. Therefore, the goal of this study was to rigorously assess both the clinical characteristics and the neuropathology of a group of individuals selected using stringent autopsy and clinical criteria for resistance or resilience specifically to ADNC (Braak stage VI and CERAD frequent) from a community-based autopsy cohort. To do this we not only implemented strict pathologic criteria for ADNC but we also performed a careful review of the rich clinical data available from the Adult Changes in Thought (ACT) study to identify participants who were cognitively intact. This unsurprisingly generated small but relatively homogeneous groups of cognitively intact outliers on either end of the ADNC spectrum which provided the opportunity to identify differences in clinical or demographic variables between groups and compared to cases with ADNC and dementia. We performed an extensive neuropathologic evaluation beyond accepted guidelines for $\mathrm{AD}$ and $\mathrm{ADRD}$ neuropathologic change, including quantitative measures of pathologic proteins in multiple brain regions and evaluation of synaptic integrity of the perforant pathway, to better characterize the tissue-level findings in these groups compared with matched participants with AD dementia. We hypothesized that non-demented subjects, identified under the most stringent criteria to remove neuropathologically borderline cases, could be discriminated from subjects with AD dementia by clinical and neuropathological traits more specific to resistance and resilience mechanisms which could be further studied in model systems. 
To that end, based on our findings from this cohort we have developed a novel experimental model of combined proteotoxicity and demonstrated synergistic toxicity of tau and TDP-43 in vivo.

\section{Materials and methods}

Adult changes in thought (ACT) study cohort

This study was approved by the Group Health (GH)/Kaiser Permanente Washington (KPW) and University of Washington (UW) Human Subjects Review Committees. The Adult Changes in Thought (ACT) study is a population-based prospective cohort study focused on brain aging and risk factors for dementia [47]. ACT is based within KPW (formerly GH), an integrated health care delivery system in Washington state. The ACT study recruits community-dwelling, nondemented adults aged 65 and older from among KP members living across the Seattle, WA area. The original ACT Study cohort included 2581 randomly selected dementia-free older adults enrolled between 1994 and 1996, and an expansion cohort $(n=811)$ was added between 2000 and 2002. In 2005, the study began ongoing enrollment to replace people who die, develop dementia, or drop out. Participants are invited to consider consenting for brain donation but consent is not required to join the study; the brain autopsy consent rate is consistently $25-30 \%$. Cognitive screening, physical function, medical history review, and functional status assessments are administered to ACT participants at study entry and subsequently every two years. Demographic and clinical characteristics are assessed during biennial ACT study visits. Overall comorbidity is measured by self-report using the Charlson Comorbidity Index, which weights comorbid diseases based on risk of mortality [16]. Cognition is measured every two years using the Cognitive Abilities Screening Instrument (CASI) [94]. CASI scores range from 0 to 100 , with higher scores indicating better cognition. Scores $\leq 85$ prompt further clinical and psychometric evaluation, and diagnoses of incident dementia are made using standardized (DSM IV and ADRDA) research criteria at a consensus conference [3]. Participants are administratively censored from the ACT cohort upon a dementia diagnosis. Dementia-free participants continue with biennial follow-up.

\section{ACT neuropathology}

A thorough neuropathology evaluation is performed for every ACT participant who provided written consent for research brain autopsy during life [50, 90]. Briefly, all neuropathologic examinations are performed in the UW Division of Neuropathology and the UW AD Research Center (ADRC) Neuropathology Core blind to clinical diagnosis. For decedents with post-mortem interval $<8$ $\mathrm{h}$, a rapid autopsy is performed in which more than 60 samples are taken from one hemisphere and flash frozen in liquid nitrogen. The remaining portion of the sampled hemisphere, and the unsampled (intact) contralateral hemisphere (or the whole brain in non-rapid cases) are evaluated for neuropathology and then immersion-fixed in $10 \%$ neutral buffered formalin for at least 2 weeks. Following fixation, all brains are evaluated (wholly and after coronal sectioning) for any gross lesions. Tissue samples are dissected from middle frontal gyrus, superior and middle temporal gyri, inferior parietal lobule, anterior cingulate gyrus, primary visual cortex, basal ganglia at the level of the anterior commissure, thalamus, hippocampus at the level of the uncus and lateral geniculate nucleus, amygdala, midbrain including substantia nigra, pons at the level of the locus ceruleus, medulla, and cerebellar hemisphere, processed, and embedded in paraffin prior to sectioning and staining. A microtome is used to cut $4 \mu \mathrm{m}$ thick tissue sections from formalin fixed paraffin embedded (FFPE) tissue blocks which are stained with hematoxylin and eosin (H\&E) and Luxol fast blue (LFB). A Bielschowsky silver stain is manually performed on $8 \mu \mathrm{m}$ thick tissue sections of mid-frontal and parietal sections. Immunohistochemistry is performed on multiple sections in alignment with the latest guidelines at the time of the autopsy. Primary antibodies used are listed in Table 1. Appropriate positive controls are included for each antibody; negative controls are also run consisting of secondary antibodies of the appropriate species in the absence of primary antibody. All ACT autopsies are evaluated by a BoardCertified neuropathologist for Braak stage, CERAD score, Lewy body disease pathology, hippocampal sclerosis, gross and microscopic (micro) infarcts, frontotemporal lobar degeneration, and all other parameters necessary for a thorough diagnostic neuropathologic

Table 1 Antibody Characteristics

\begin{tabular}{llllllll}
\hline Target & Clone & Raised in & Poly v. Mono & Company & Dilution & Pretreatment & Brain regions \\
\hline B-amyloid & 6E10 & Mouse & Mono & Covance & 1:2500 & ER2 (20 min) & MFG, MTG, STG, IPL, OCX, striatum, \\
& hippocampus, MB, CBL & & & & \\
PHF-tau & AT8 & Mouse & Mono & Pierce & $1: 1000$ & ER 1 (10 min) & MFG, MTG, STG, IPL, OCX, hippocampus, \\
a-synuclein & LB509 & Mouse & Mono & Invitrogen & $1: 250$ & ENZ1 (10 min) & MFG, IPL, amygdala, MB \\
pTDP-43 & ID3 & Rat & Mono & Millipore & 1:1000 & ER 1 (10 min) & MFG, MTG, STG, amygdala, hippocampus \\
Synaptophysin & MRQ-40 & Rabbit & Mono & Novus Biologicals & 1:100 & ER 2 (20 min) & MFG, MTG, STG, OCX, hippocampus \\
\hline
\end{tabular}


exam. Starting in 2012, all ACT autopsies were evaluated according to the NIA-AA guidelines for assessment of ADNC and neuropathology of related disorders [55]; thus, Thal phase scores are not available in routine ACT autopsies that predate 2012. Phospho-TDP-43 (pTDP43) pathology assessment was not standardized until 2013. Recognizing the challenges of an evolving neuropathology dataset, existing AD neuropathological criteria common to every ACT autopsy (Braak, CERAD) were used for case selection (see below) and then a combined qualitative and quantitative neuropathology evaluation was performed on every case under the same conditions (see below) to better understand differences between resistance/resilience and AD dementia.

\section{Case selection}

Cases were selected for this study based on their cognitive status during life and the degree of ADNC as assessed at autopsy. Only neuropathologic data that exists for all ACT autopsies were used for case selection, specifically, Braak stage and CERAD score. The resistant group included all non-demented (CASI within two years of death) ACT participants with Braak stage III or lower and CERAD none who were age 85 years or older. Using these criteria, we identified 14 subjects out of the 684 in the ACT autopsy cohort. The resilient group included all non-demented (CASI within two years of death) ACT participants with neuropathologically verified severe ADNC (defined as Braak stage VI, CERAD frequent). Using these criteria, we identified 7 subjects in the ACT autopsy cohort. AD dementia cases for both resistant and resilient groups were matched in a one-toone algorithm that linked each index case with same sex, demented (based on clinical evaluation including psychometric testing and consensus agreement) participants with neuropathologically confirmed severe ADNC (Braak stage VI, CERAD frequent). Age at death and year of death were used to identify the best match from the linked set for each case. If more than one matched case was still available, post-mortem interval closest to the index case was used to select the best match, which was then removed from the pool of cases available for the matching algorithm for subsequent cases.

\section{Standardized Neuropathologic assessment Gross neuropathological exam}

As part of the ACT autopsy cohort, every brain (including bilateral cerebrum, cerebellum, and brainstem) had undergone a detailed gross neuropathological analysis including assessment for cerebral cortical atrophy. The degree of atherosclerosis was also noted based on a four-point scale (absent, mild when restricted to branch points in the circle of Willis, moderate when also in other regions at the base of the brain, and severe when present on the convexity of cerebrum). Sampling of bilateral cerebral, brainstem, and cerebellar structures was performed for every case (22 routine samples per brain). Any suspected chronic (cystic) territorial and lacunar infarcts were recorded, measured, and additionally sampled for histopathological examination; the total number of gross infarcts for each case was then recorded. Any other focal lesions, including hemorrhage, mass lesions, and other abnormalities were sampled.

\section{Ordinal/semi-quantitative assessment}

After resistant, resilient, and $\mathrm{AD}$ dementia matched groups were selected based on the existing Braak and CERAD data, we controlled for variations of staining reagents, techniques, and neuropathological interpretation over time by performing an additional and extensive evaluation for neurodegenerative disease on each case based on the 2012 NIA-AA criteria [55]. This involved cutting and staining the original FFPE blocks from diverse regions according to NIA-AA recommendations; additional dissections in wet tissue were not required as the historic routine diagnostic sampling in ACT aligned with the NIA-AA guidelines. We used the newly stained sections to evaluate ADNC for each case; we classified neurofibrillary degeneration by characterizing neurofibrillary tangle distribution across medial temporal, isocortical, and primary sensory cortex by the method of Braak and Braak [11, 12], scored neuritic plaque density in isocortex by the method of the Consortium to Establish a Registry for Alzheimer's Disease staging [CERAD] $[53,54]$, and assessed $A \beta$ plaque distribution as described by Thal [95]. pTDP-43 pathology was evaluated in amygdala, hippocampus, temporal and frontal cortex similar to published protocols $[66,101,103]$. We characterized Lewy body disease using routine and immunohistochemical stains in the brainstem, amygdala, anterior cingulate gyrus, and frontal (middle frontal gyrus) and parietal (inferior parietal lobule) cortex according to current guidelines [22] and performed a thorough evaluation for chronic macroscopic and microscopic vascular brain injury. For microvascular brain injury, samples of bilateral middle frontal gyrus, inferior parietal lobule, super/middle temporal gyrus, and medial occipital lobe including calcarine cortex, as well as bilateral neostriatum (at the level of the anterior commissure) and thalamus, were sampled for histologic analysis using hematoxylin and eosin combined with Luxol fast blue (H\&E/LFB) stains, and the number of cerebral microinfarcts was recorded for these standardized screening sections based on published protocols [31, 55, 90, 98]. Arteriolosclerosis was assessed on a four-point scale (absent, mild when perivascular hemosiderin was present and walls were slightly thickened, moderate when arteriole walls were markedly thickened, and severe when 
there was luminal narrowing with prominent lamination (onion-skinning) of the vessel wall).

\section{Summary neuropathology score}

A summary neuropathology score was created as a descriptive metric to capture the relative magnitude of $A D$, $\mu \mathrm{VBI}, \mathrm{LBD}$, and limbic-predominant age-related TDP-43 encephalopathy neuropathologic change (LATE-NC) in each individual. Similar to previously published scores $[56,92]$, this summary neuropathology score derives from the sum of subscores for each of the main axes: (i) ADNC subscore (Braak stage for NFTs expressed as a number and then divided by $2(0-3)$ plus CERAD score expressed as a number (0-3), and thus ranging from 0 to 6), (ii) $\mu \mathrm{VBI}$ subscore (number of chronic microinfarcts (CMI) with $\geq 3 \mathrm{CMI}$ by screening protocol assigned a value of 3 and thus ranging from 0 to 3), (iii) LBD subscore ( 0 for none, 1 for brainstem LBD, 2 for limbic or amygdala-only LBD, and 3 for isocortical LBD in frontal or parietal cortex), and LATE-NC subscore $(0$ for none, 1 for amygdala only, 2 for hippocampal, and 3 for isocortical in frontal or temporal cortex).

To extend assessment of ADNC beyond NIA-AA guidelines, the local burden of pTau and $A \beta$ plaque pathology was recorded from multiple brain regions on a three-point scale. All assessments were performed by a neuropathologist blinded to dementia diagnosis and original ADNC classification. Hyperphosphorylated tau (pTau) was evaluated using the AT8 antibody scored as: $0=$ no tangles, $1=$ rare neurofibrillary tangles $(<3$ per $10 x$ field) with sparse neurites, 2 = numerous tangles (310 per $10 x$ field) and scattered neurites, and $3=$ extensive tangle pathology ( $>10$ per $10 \mathrm{x}$ field) with abundant neurites. $\mathrm{A} \beta$ was evaluated using the $6 \mathrm{E} 10$ antibody and scored as: $0=$ no plaques, $1=$ rare plaques $(<5$ per $10 \mathrm{x}$ field), 2 =numerous plaques $(5-20$ plaques per $10 \mathrm{x}$ field), and 3 =extensive plaques ( $>20$ per $10 x$ field). Cerebral amyloid angiopathy was graded on a four-point scale $(0=$ absent, $1=$ mild when restricted to the leptomeningeal vessels, 2 = moderate when involving a minority of penetrating arterioles, and $3=$ severe when involving a majority of penetrating arterioles) in the frontal, parietal, temporal, and occipital lobes as well as the cerebellum. In addition to assessment of pTDP-43 pathology distribution, in each region a score of 0-6 was assigned based on the density of pTDP-43 pathology (combined inclusions and neurites) in a 20x field $(0=$ none, $1=1-2$ inclusions/neurites, $2=3-5$ inclusions/ neurites, $3=6-10$ inclusions/neurites, $4=11-15$ inclusions/neurites, $5=15-20$ inclusions/neurites, and $6=>$ 20 inclusions/neurites. Severity of microvacuolation and gliosis, a measure of neurodegenerative change, was assessed in H\&E/LFB stained sections on a four-point scale $(0=$ absent, $1=$ mild when limited to superficial cortical layers I and II, 2 = moderate when extending to layers III and IV, and 3 = severe, when extending through all cortical layers to the subcortical white matter) for each brain region examined.

\section{Quantitative neuropathology using digital slide scanning}

Sections immunostained for synaptophysin, pTDP-43, and pTau were scanned using an Aperio ScanScope AT2 (Leica Biosystems Pathology Imaging, Vista, CA) at 20x $(0.5 \mu \mathrm{m} /$ pixel $)$, and stored on a server running Aperio eSlide Manager digital slide repository and database software. Using Aperio ImageScope software (Leica Biosystems Pathology Imaging, Vista, CA), each tissue section was annotated with the freeform pen tool to create regions of interest (ROI) for analysis. Quantitative image analysis of the annotated ROIs was performed using Aperio Brightfield Image Analysis Toolbox software (Leica Biosystems Pathology Imaging, Vista, CA). The RGB color vectors of the blue hematoxylin counterstain (R:0.67, G:0.66, B:0.339) and brown DAB chromogen (R: 0.311, G:0.522, B:0.794) were measured using the default Color Deconvolution algorithm in areas where only one of the stain components was present. A third residual component (R:0.02, G:0.999, B:0.02) was used to optimize digital stain separation. The calibrated RGB color vectors were input as parameters into the Color Deconvolution Area Analysis algorithm (pTau stained slides) and IHC Nuclear Quantification algorithm (pTDP-43+ stained slides) to provide digital separation of staining components and allow for quantification of the positive chromogen in the separated DAB channel.

The IHC Nuclear Quantification algorithm was calibrated to count the number of pTDP-43+ inclusions per annotated area. This algorithm finds positively stained objects based on size, shape, and positive intensity threshold parameters. The object segmentation properties were set to use a smoothing radius $(2 \mu \mathrm{m})$, merging threshold for declustering (2.5), and a manual threshold value (220) of the DAB channel intensity by setting the threshold to the highest possible value and reducing it until background and non-specific staining were removed from the segmented areas. Object shape and size restrictions were set for minimum size $\left(20 \mu \mathrm{m}^{2}\right)$, maximum size $\left(225 \mu \mathrm{m}^{2}\right)$, minimum roundness $(0.25)$, and minimum elongation (0.1). The upper positive intensity threshold for pTDP-43+ objects was calibrated (value 200) by running the above parameters on a training set of 5 positive slides and 5 negative slides. Histogram data of the number of objects per intensity unit was extracted and the cumulative frequencies were used to calculate the final output metric (objects per $\mathrm{mm}^{2}$ ) at each possible threshold cut point. The performance of the algorithm was examined in the positive and negative slides, and the positive threshold was selected at the point 
where the number of objects per $\mathrm{mm}^{2}$ reached a maximum on the positive slides while remained minimum on the negative slides (99th percentile of objects in positive slides, 60th percentile in negative slides). The Color Deconvolution Area Analysis algorithm was calibrated to measure the total amount of staining present in the pTau ROIs. This algorithm counts the number of pixels which exceed an upper intensity threshold in the DAB stain channel. The upper positive intensity threshold (200) was calibrated by measuring the performance of the algorithm on a training set of 5 positive slides and 5 negative slides. Histogram data of the positive area per intensity unit was extracted and the cumulative frequencies were used to calculate the final output metrics (percent positive and average positive optical density) at each possible threshold cut point. The performance of the algorithm was examined in the positive and negative slides, and the positive threshold was selected at the point where the output metrics for the positive slides were greater than the metrics for the negative slides. Some non-specific signal was observed in naturally pigmented lipofuscin and rare hemosiderin regions. All other algorithm values were left as default.

The quantitative analysis data for each ROI were extracted into Microsoft Excel for further analysis. The data included total numbers of pTDP-43+ objects per $\mathrm{mm}^{2}$ and for pTau the product of average positive optical density (OD) multiplied by percent of ROI with positive staining (OD*\% Positive). Optical density is a measurement of absorbance and is linearly related to the amount of staining present [46]. OD*\%Positive is a weighted metric previously used in digitally quantifying immunohistochemical staining $[10,76,77]$.

\section{Relative Immunointensity ratio}

To determine differences in hippocampal integrity, synaptic density in the perforant pathway was measured using synaptophysin-immunostained hippocampal sections. Synaptophysin optical density of the outer molecular layer of the hippocampus was measured in relation to that of the inner molecular layer, generating a relative immunointensity ratio (RIR) similar to previously published methods [80]. ImageJ 1.452j (National Institutes of Health) was used for analysis. Mean pixel intensities of the inner and outer molecular layer of the dentate gyrus just under CA1 were measured. Raw values on an 8-bit scale were normalized to a blank area for each slide. Synaptic RIRs of the outer/inner molecular layer were calculated from (outer molecular layer blank) / (inner molecular layer - blank) for each case.

\section{C. elegans}

\section{Strains and transgenics}

Wild-type C. elegans (Bristol strain N2) was maintained as previously described [13]. Transgenic strains used were CK410 bkIs410[Psnb-1::hTDP-43 + Pmyo-2:: dsRED], CK491 bkIs491[Psnb-1::GFP + Pmyo-2::dsRED], CK1044 bkIs1044[Paex-3::tau(WT 4R1N) + Pmyo-2:: GFP] [51, 93].

\section{Analysis of transgene segregation and synthetic lethality analysis}

Parent C. elegans homozygous for human (h) Tau tg (CK1044) but heterozygous for the other transgene of interest (Tg control (CK491) or hTDP-43 tg (CK410)) were generated using a standard mating cross of the two strains, and scoring F2 progeny (F3 animals) for the desired genotype (F2 genotype: htau tg $+/+$, other transgene $+/-)$. Every progeny from a single htau tg $+/+$; other tg $+/$ - parent was then singled blind with regards to transgene status onto individual plates with food, and their progeny scored for the transgene of interest $(\mathrm{Tg}$ control (CK491) or hTDP-43 tg (CK410). This was performed in triplicate for each transgene of interest.

\section{Behavioral analysis}

Assessments of C. elegans locomotion were carried out as previously described with minor modifications [51]. In brief, 15-20 animals were placed at the center of a $100 \mathrm{~mm}$ plate of $5 \mathrm{x}$ concentrated peptone nematode growth media uniformly seeded with OP-50 bacteria. Animals were allowed to move freely for $30 \mathrm{~min}$, and the radial distance traveled from the start point was recorded. Distance traveled was converted to micrometers per minute.

\section{Immunoblotting}

Stage-matched day 1 adult $C$. elegans were harvested and snap frozen. Protein was extracted by resuspending pellets in $1 \mathrm{X}$ sample buffer, $20 \mathrm{~s}$ sonication, and $5 \mathrm{~min}$ boiling. $10 \mu \mathrm{L}$ of samples were loaded onto precast 4$15 \%$ gradient TGX (tris glycine stain-free) gels (Bio-Rad) and electroblotted to PVDF membrane (Bio-Rad). Membranes were blocked in 5\% milk; total TDP-43 was detected with mouse monoclonal antibody ab57105 (Abcam) and phosphorylated TDP-43 (pS409/410) was detected with mouse monoclonal antibody TIP-PTDM01 (CosmoBio). Total tau was detected with Rb17025 antibody (generous gift of Dr. V.M. Lee) and pTau was detected with AT180 (pT231, Thermo Scientific). betaTubulin antibody E7 (DSHB) was used as a load control for all samples.

\section{Analysis/statistics}

For demographic and clinical data, binary variables were compared using McNemar's chi-square test with exact $p$-values, while continuous variables were compared using the Wilcoxon matched-pairs signed-ranks test. We used Wilcoxon matched-pairs signed-ranks tests for 
analysis of all neuropathology variables. We used Stata (Version 15.1, StataCorp, College Station, Texas) for all analyses. Analysis of genetic distribution and locomotion in $C$ elegans experiments was calculated by comparing expected Mendelian genetic ratios for one segregating trait and analyzed by Chi square analysis (GraphPad Prism 7, San Diego, CA).

\section{Results}

\section{Demographic and clinical characteristics}

We anticipated our stringent selection criteria would lead to a small number of cases, which was indeed the case. Of the $684 \mathrm{ACT}$ autopsies screened for this study, we identified 14 resistant cases and 7 resilient cases using existing ACT neuropathology criteria. CASI score was used as a primary selection criterion for cases with dementia, and a two-year cutoff was assigned in alignment with prior studies [92, 99]. We then evaluated for each cohort the range of CASI latencies prior to death, with the assumption that a shorter latency provides a higher degree of confidence in the cognitive assessment. We found that the resistant group as a whole had a mean CASI latency of 1.04 years, with a range from 92 days to 1.8 years, and half having a CASI latency of less than one year. The resilient group had a mean CASI latency of 316 days, with a range from 61 days to 1.6 years; with four of the seven cases having a latency of less than one year. Although a final cognitive test within one year of death is desirable, and was achieved in roughly half of the individuals in this study, prior studies have demonstrated the relative reliability of using a two-year cutoff $[92,99]$. Most resistant and resilient individuals maintain relatively stable cognitive function as assessed on CASI score across their time involved in the ACT study. Figure 1 illustrates the limited decline in the majority of resistant and resilient subjects over time.

There were not significant differences in most demographics (see Table 2). With respect to genotype, as might be expected, a smaller proportion of the resistant group had $\geq 1 A P O E \varepsilon 4$ allele than the matched AD dementia participants $(p=0.03)$, but the proportion of people with $\geq 1$ APOE $\varepsilon 4$ allele was not statistically different between resilient participants and their AD dementia matches. The mean number of years of formal education was greater for the resistant group compared to their AD dementia matches (15.1 vs. 13.2 years, respectively), but the difference was not statistically significant $(p=0.10)$. Differences in education levels were not detected between resilient cases and AD dementia matches. These findings are summarized in Table 2.

\section{Standard neuropathology $A D$ Neuropathologic change}

All cases underwent a standardized neuropathologic evaluation using current guidelines and common immunostains, which resulted in reclassification of some cases. Specifically, six cases (one resilient, one AD dementia match for the resilient group, and four AD dementia matches for the resistant group) were reclassified from Braak stage VI to Braak stage V based on the absence of neurofibrillary tangles in calcarine cortex without considering dentate gyrus neurofibrillary tangle burden, which may be less exclusive to Braak stage VI. In the resistant group, seven cases had more widely distributed neurofibrillary tangle pathology using AT8 pTau stains rather than historical (for ACT) Tau2 immunostaining, including some cases with rare cerebral cortex (mostly middle temporal gyrus) tangles and were reclassified (upward) as Braak stage IV [11]. One case in the resistant group was re-classified as a CERAD sparse due to the identification of rare neuritic plaques in the cortex identified by PHF-tau immunostain (AT8). No cases were reclassified more than one level of Braak stage or CERAD score. After reclassification, the resistant group still had lower levels of AD pathology compared to their $\mathrm{AD}$ dementia matches (as expected, given the selection criteria), while the resilient group and their $\mathrm{AD}$ dementia matches showed similar degrees of ADNC as assessed by Thal phase, Braak stage and CERAD score, verifying that historical misclassification was not an underlying driver of resistance/resilience in this group. These findings are illustrated in Fig. 2.

\section{Other Neuropathologic findings}

For each case, additional neuropathologic findings were assessed, including the presence and extent of Lewy body disease (LBD) and limbic-predominant age-related TDP-43 encephalopathy (LATE), and evidence of vascular brain injury (VBI), including degree of arteriolosclerosis and atherosclerosis, the number of macro- and microinfarcts, and the severity of cerebral amyloid angiopathy. Overall, the most striking difference between participants without dementia (both resistant and resilient) and their AD dementia matches was the absence of LATE neuropathologic change (LATE-NC) $(p=0.0009$, resistant and $p=0.047$, resilient). pTDP-43 pathology was not considered in selecting cases and matches (and wasn't available for most of the cohort), yet in the resistant group, any pTDP-43 pathology was identified in only 2 of 14 subjects, significantly different from their $\mathrm{AD}$ dementia matches where pTDP-43 pathology was present in 13 of 14 cases. In the resilient group a single subject was found to have pTDP-43 pathology, while all but one of the AD dementia matches exhibited pTDP-43 pathology that extended at least to involve the hippocampus. This is remarkable considering pTDP-43 was not part of selection criteria for this study and suggests LATE-NC may be much more prevalent than indicated by historical neuropathological characterization in the ACT study. 


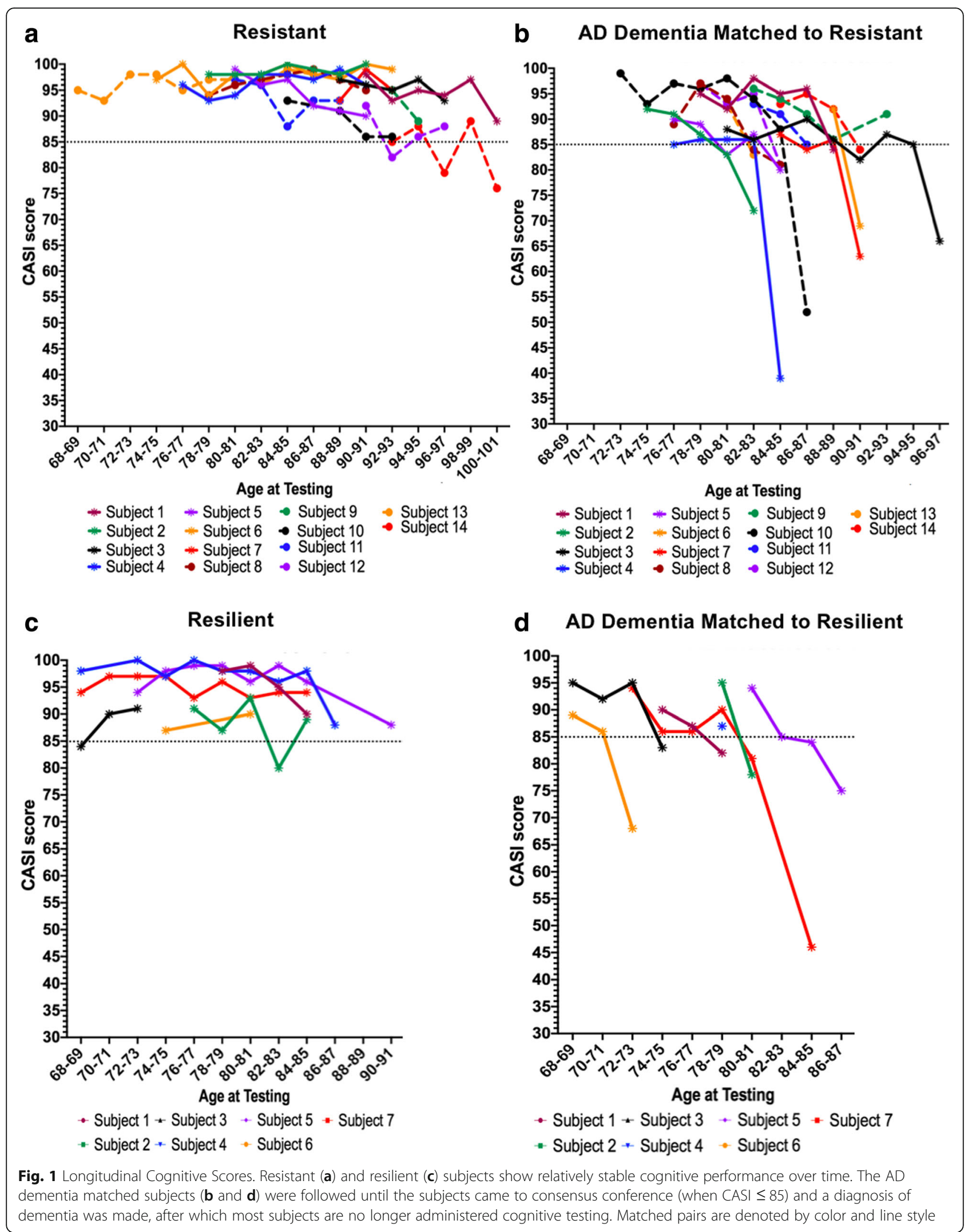


Table 2 Clinical characteristics by resistance and resilience status

\begin{tabular}{|c|c|c|c|c|c|c|}
\hline & $\begin{array}{l}\text { Resistant } \\
(n=14)\end{array}$ & $\begin{array}{l}\text { AD Dementia } \\
\text { Matched to Resistant } \\
(n=14)\end{array}$ & $p$ value & $\begin{array}{l}\text { Resilient } \\
(n=7)\end{array}$ & $\begin{array}{l}\text { AD Dementia } \\
\text { Matched to Resilient } \\
(n=7)\end{array}$ & $p$ value \\
\hline $\begin{array}{l}\text { Age at death (years) } \\
\text { mean (SD) }\end{array}$ & 94.7 (3.8) & $94.5(2.5)$ & 0.47 & $85.2(6.1)$ & 86.7 (3.5) & 0.31 \\
\hline $\begin{array}{l}\text { Sex - female } \\
\mathrm{n}(\%)\end{array}$ & $8(57.1)$ & $8(57.1)$ & 1.00 & $5(71.4)$ & $5(71.4)$ & 1.00 \\
\hline $\begin{array}{l}\text { Education (years) } \\
\text { mean (SD) }\end{array}$ & $15.1(3.1)$ & $13.2(2.6)$ & 0.10 & $15.9(2.7)$ & $15.4(3.7)$ & 0.67 \\
\hline $\begin{array}{l}A P O E, \mathrm{n}(\%) \\
\varepsilon 2 / 2 \text { allele }\end{array}$ & $0(0.0)$ & $2(14.3)$ & & $0(0.0)$ & $0(0.0)$ & \\
\hline$\varepsilon 2 / 3$ allele & $2(14.3)$ & $0(0.0)$ & & $1(14.3)$ & $0(0.0)$ & \\
\hline ع3/3 allele & $11(78.6)$ & $5(35.7)$ & & $4(57.1)$ & $2(33.3)$ & \\
\hline ع2/4 allele & $0(0.0)$ & $0(0.0)$ & & $0(0.0)$ & $1(16.7)$ & \\
\hline ع3/4 allele & $1(7.1)$ & $7(50.0)$ & & $2(28.6)$ & $3(50.0)$ & \\
\hline $\begin{array}{l}1 \text { copy of } \varepsilon 4 \text { allele } \\
\mathrm{n}(\%)\end{array}$ & $1(7.1)$ & $7(50.0)$ & 0.03 & $2(28.6)$ & $4(66.7)$ & 0.50 \\
\hline $\begin{array}{l}\text { Age at final study visit (years) } \\
\text { mean (SD) }\end{array}$ & 93.1 (3.9) & $87.4(3.7)$ & $<0.001$ & 83.9 (5.6) & 79.7 (5.5) & 0.05 \\
\hline $\begin{array}{l}\text { CASI to death (years) } \\
\text { mean (SD) }\end{array}$ & $1.0(0.6)$ & $6.8(2.8)$ & 0.001 & $0.9(0.5)$ & $7.0(3.4)$ & 0.03 \\
\hline $\begin{array}{l}\text { Charlson Comorbidity Index } \\
\text { (cumulative) mean (SD) }\end{array}$ & $2.2(1.9)$ & $2.1(2.0)$ & 0.90 & $3.4(2.6)$ & $1.1(1.3)$ & 0.06 \\
\hline $\begin{array}{l}\text { Smoking (cumulative pack years) } \\
\text { mean (SD) }\end{array}$ & $14.0(21.1)$ & $14.8(16.3)$ & 0.43 & 19.3 (21.6) & $4.6(9.6)$ & 0.15 \\
\hline
\end{tabular}

Wilcoxon matched-pairs signed-ranks test. Note: Cumulative measures were based on self-report at the last ACT study visit attended. Participants diagnosed with dementia were administratively censored from ACT, whereas dementia-free participants continued with biennial study visits. Bold values indicate statistical significance

We found significant differences between the resistant participants and their AD dementia matches with respect to brain weight (1228 g, $1115 \mathrm{~g} ; p=0.0011)$, burden of cerebral amyloid angiopathy $(0.50,1.36 ; p=0.015)$ and arteriolosclerosis $(2.00,2.57 ; p=0.031$ ) (for details see Table 3). Atherosclerosis was lower on average in resistant people but this difference was not significant
(1.93, 2.29; $p=0.097)$. In the resilient group, chronic macroinfarcts were not identified in resilient subjects but were present in over half of matched AD dementia subjects $(0,0.86 ; p=0.048)$. All neuropathology variables are listed, with means, SD, and $p$ values, in Table 3. Statistical analysis was not performed for Braak stage, CERAD score, and Thal phase because Braak and
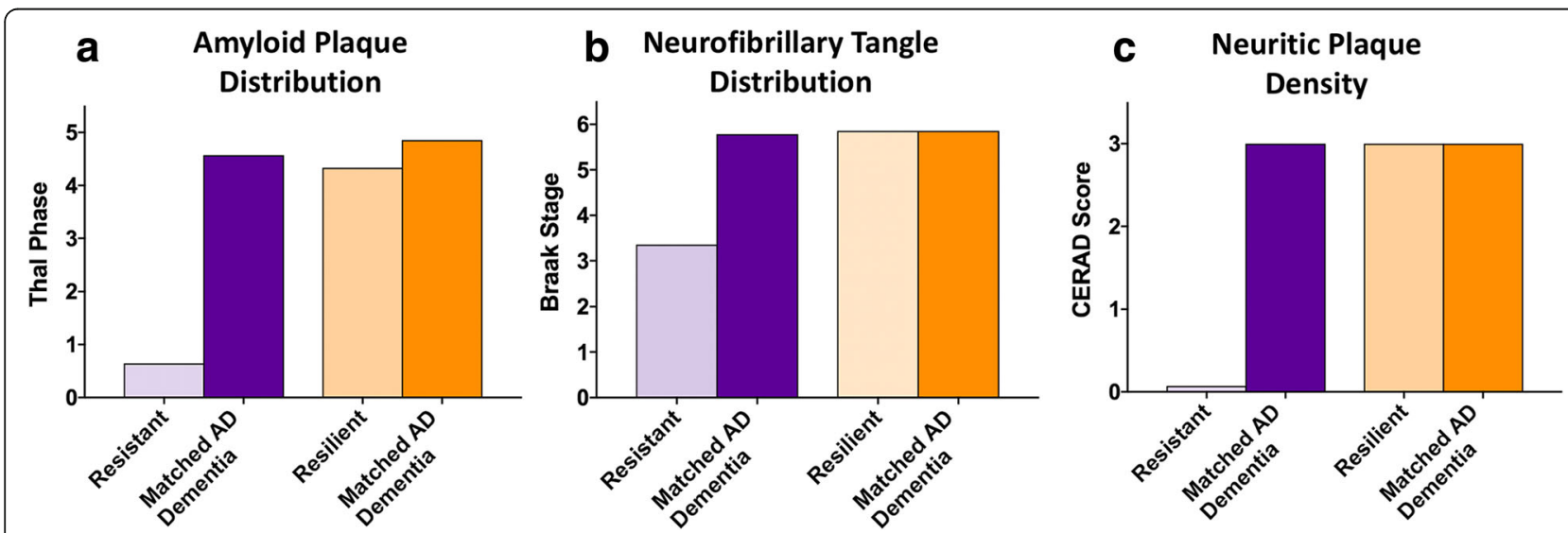

Fig. 2 AD Neuropathologic change. Cases were selected based on the degree of AD neuropathologic change. The resistant cases were selected based on a Braak score of III or less and absent neuritic plaques by CERAD criteria. Their AD dementia matches, the resilient cases, and their respective $\mathrm{AD}$ dementia matches were selected based on a Braak stage $\mathrm{VI}$ and frequent neuritic plaques. Re-evaluation demonstrated the expected distribution of amyloid plaques (a), neurofibrillary tangles (b), and density of neuritic plaques (c), based on these selection criteria 
Table 3 Neuropathologic characteristics by resistance and resilience status

\begin{tabular}{|c|c|c|c|c|c|c|}
\hline $\begin{array}{l}\text { Pathology } \\
\text { (mean+/-SD) }\end{array}$ & $\begin{array}{l}\text { Resistant } \\
(\mathrm{n}=14)\end{array}$ & $\begin{array}{l}\text { AD Dementia Matched to Resistant } \\
(\mathrm{n}=14)\end{array}$ & $p$ value & $\begin{array}{l}\text { Resilient } \\
(\mathrm{n}=7)\end{array}$ & $\begin{array}{l}\text { AD Dementia Matched to Resilient } \\
(\mathrm{n}=7)\end{array}$ & $p$ value \\
\hline Brain Weight (grams) & $1228(75)$ & $1115(84)$ & 0.001 & $1223(151)$ & $1120(205)$ & 1.128 \\
\hline Thal Phase & $0.64(0.50)$ & $4.57(0.51)$ & ND & $4.33(0.52)$ & $4.86(0.38)$ & ND \\
\hline Braak Stage & $3.36(0.74)$ & $5.79(0.43)$ & ND & $5.86(0.38)$ & $5.86(0.38)$ & ND \\
\hline CERAD score & $0.07(0.27)$ & $3.00(0.0)$ & ND & $3.00(0.0)$ & $3.00(0.0)$ & ND \\
\hline Atherosclerosis & $1.93(0.62)$ & $2.29(0.50)$ & 0.097 & $1.86(1.10)$ & $1.57(0.53)$ & 0.421 \\
\hline Arteriolosclerosis & $2.00(0.68)$ & $2.57(0.51)$ & 0.031 & $2.14(0.38)$ & $2.29(0.76)$ & 0.655 \\
\hline Macroinfarcts & $0.50(0.85)$ & $0.57(0.76)$ & 0.895 & $0(0)$ & $0.86(1.07)$ & 0.048 \\
\hline Microinfarcts & $1.07(1.27)$ & $1.50(1.23)$ & 0.356 & $0.57(1.13)$ & $1.14(1.07)$ & 0.389 \\
\hline CAA severity (OC) & $0.50(0.85)$ & $1.36(0.84)$ & 0.015 & $1.86(1.35)$ & $1.42(0.79)$ & 0.252 \\
\hline Lewy body distribution & $1.00(1.57)$ & $1.50(1.83)$ & 0.388 & $0(0)$ & $0.57(1.51)$ & 0.317 \\
\hline LATE-NC & $0.21(0.58)$ & $2.40(0.94)$ & 0.0009 & $0.43(1.13)$ & $1.86(0.90)$ & 0.047 \\
\hline
\end{tabular}

Wilcoxon matched-pairs signed-ranks test; ND, Not Done; LATE-NC, limbic-predominant age-related TDP-43 encephalopathy neuropathologic change. Bold values indicate statistical significance

CERAD were used as selection criteria, and Thal may be considered directly related to CERAD.

\section{Summary neuropathology score}

Summary neuropathology scores were evaluated for all subjects to represent overall $\mathrm{AD}$ and ADRD pathologic burden and are represented graphically in Fig. 3. Each subject's summary neuropathology score is a bar divided into its corresponding subscores for ADNC (grey), $\mu \mathrm{VBI}$ (slate), LBD (black), and LATE-NC (red); these data have been arranged by summary neuropathology score (lowest to highest), then ranked by AD subscore, followed by $\mu \mathrm{VBI}$ subscore, and then by LBD subscore. These results highlight the findings that it is extremely uncommon for non-demented subjects (whether resistant or resilient to $\mathrm{AD}$ pathology) to exhibit LATE-NC, and resistant and resilient cases have less co-morbid neuropathology burden compared with matched AD demented subjects.

\section{Semi-quantification}

Regional semi-quantitative measurements were made for A $\beta$ (3-point scale), pTau (3-point scale) and pTDP-43 (6-point scale). As expected, there were differences in both $\mathrm{A} \beta$ and pTau in resistant individuals compared to AD dementia matches in every brain region assessed (Fig. 4). pTDP-43 was also less abundant in the resistant group in multiple brain regions (amygdala, 0.57, 4.29; $p=0.002$; hippocampus, $0.43,2.71 ; p=0.012$; middle temporal gyrus, $0.0,2.0 ; p=0.006$; superior temporal gyrus, $0.0,1.86, p=$ $0.009)$. In the resilient group, we found no difference in cortical $\mathrm{A} \beta$ burden compared to $\mathrm{AD}$ dementia matches; however, at higher Thal phases the resilient group had less amyloid on average than their dementia matches (midbrain, 1.57, 2.71; $p=0.030$; cerebellum, 0.57, 1.29; $p=0.025$ ). Although resilient cases were matched to $\mathrm{AD}$ dementia cases by Braak stage, there was significantly less pTau in the middle frontal gyrus of the resilient group (1.29, 2.57; $p=0.037$ ). We also found significantly less pTDP-43 in the amygdala $(0.14,4.29 ; p=0.028)$ and the hippocampus $(0.0,2.71$; $p=0.017)$. These results are summarized in Fig. 4 .

\section{Microvacuolation}

In addition to assessing each case for pathologic peptide deposition, we wanted to understand the parenchymal injury associated with dementia, so each cortical lobe in every case was evaluated by H\&E/LFB stain for the degree of neurodegenerative change as characterized by the severity of microvacuolation and gliosis. We found significantly less neurodegenerative change in the resistant cases in multiple cortical regions, including inferior parietal lobule $(1.00,1.71 ; p=0.026)$, middle temporal gyrus $(1.21,1.93 ; p$ $=0.037)$ and occipital cortex $(0.79,1.43 ; p=0.018)$ when compared to $\mathrm{AD}$ dementia matches. There was also less neurodegenerative change on average in the middle frontal gyrus and superior temporal gyrus, but these differences were not statistically significant $(1.07,1.43 ; p=0.059$ and $1.07,1.71 ; \mathrm{p}=0.059$, respectively). While these findings may be expected based on selection criteria, we found even stronger relationships of neurodegenerative change and dementia in the resilient group, where every cortical region had significantly less neurodegenerative change than their $\mathrm{AD}$ dementia matches (each at $p<0.05$ ). These results are summarized in Fig. 5.

\section{Quantitative neuropathology pTau and pTDP-43 burden}

Quantitative analysis of pathologic burden using analysis of imaged immunostained sections was performed for pTau across diverse brain regions, including transentorhinal cortex, hippocampus, entorhinal cortex, mesial 


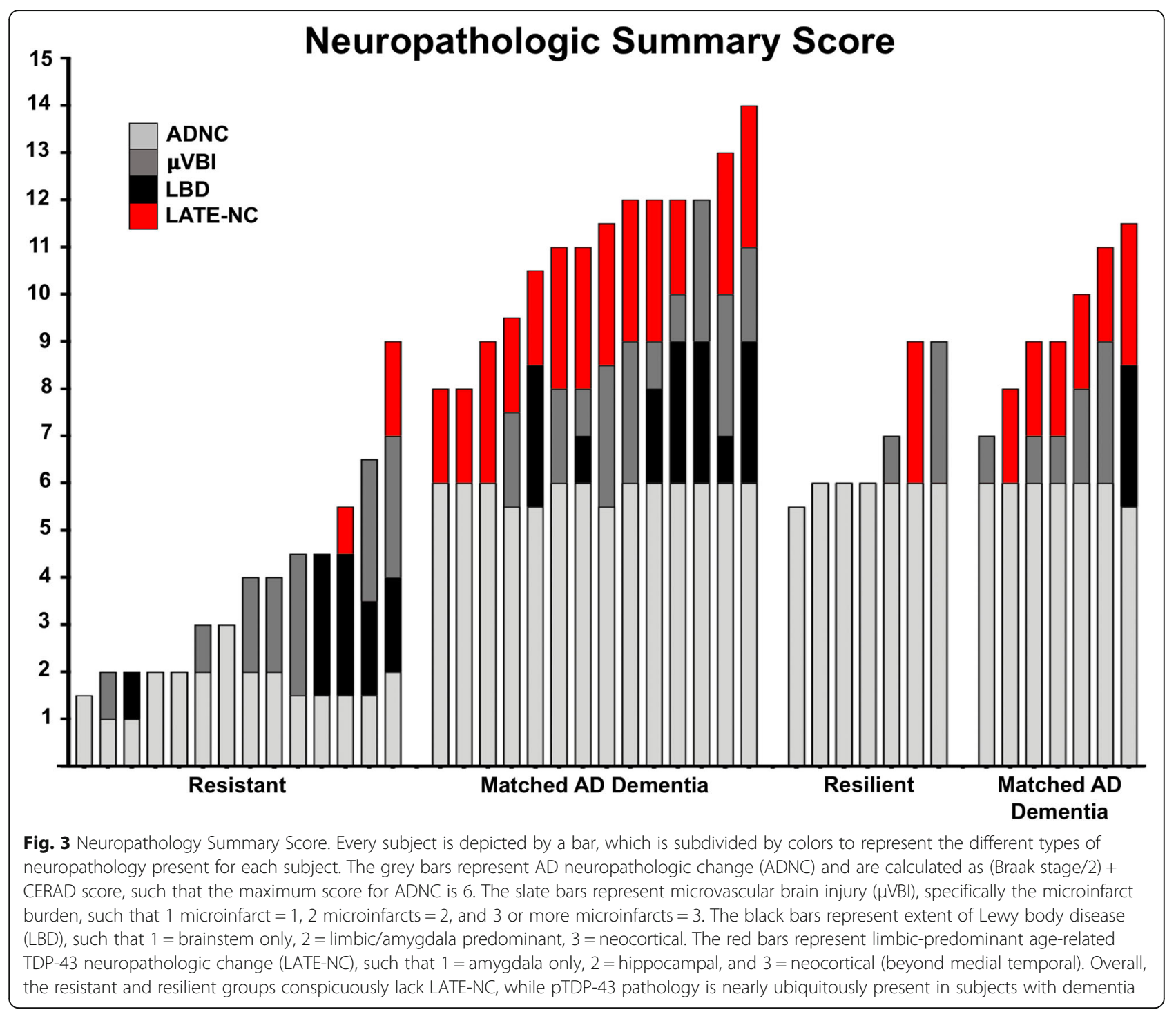

temporal cortex, middle temporal gyrus, superior temporal gyrus, and middle frontal gyrus, and for pTDP-43 in amygdala, transentorhinal cortex, entorhinal cortex, hippocampus, mesial temporal cortex, middle temporal gyrus, superior temporal gyrus, and middle frontal gyrus. Analysis of each region of interest (ROI) included a measure of optical density (OD) of the chromogen in positive areas for pTau and a count of positive objects for pTDP-43. As expected, the resistant group had less pTau immunoreactivity on average in all regions assessed compared to the matched AD dementia group (Fig. 6a). There was less pTDP-43 in the resistant group on average in the amygdala $(1.15,9.61 ; p=0.0030)$, hippocampus (0.70, 2.13; $p=0.016)$, entorhinal cortex $(0.58,1.70$; $p=0.048)$, transentorhinal cortex $(0.90 .7 .25 ; p=0.016)$, mesial temporal cortex $(0.31,3.76 ; p=0.028)$, and middle temporal gyrus $(0.35,3.02 ; p=0.011)$ (Fig. 6b).
In the resilient group, there was significantly less pTau burden in the middle frontal gyrus on average compared to the matched AD dementia group (1.07, $8.63 ; p=0.043)$ but no significant differences were detected in the other brain regions evaluated (Fig. 7a), suggesting the critical point of differentiation between normal cognition and dementia involves pTau burden, at least in part, in frontal cortex. The resilient group had lower levels of pTDP-43 burden on average compared to the matched $\mathrm{AD}$ dementia group in the hippocampus $(0.42,1.60 ; p=0.042)$, entorhinal cortex $(0.33,2.28 ; p=0.028)$, and transentorhinal cortex $(0.30,6.04 ; p=0.042)$, while we found no significant differences between resilient and $\mathrm{AD}$ dementia matched individuals in amygdala, mesial temporal cortex, middle or superior temporal gyri, or middle frontal gyrus (Fig. 7b). 







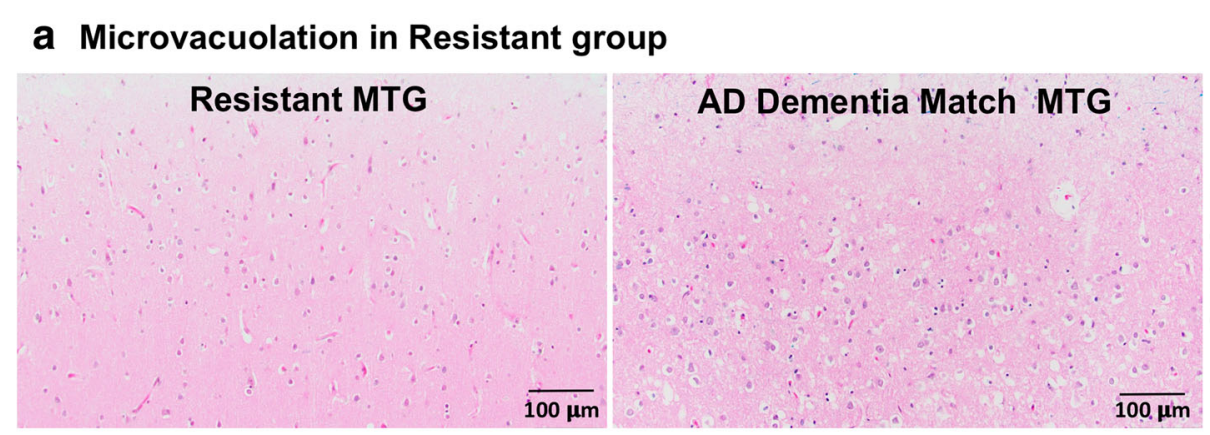

\section{b Microvacuolation in Resilient group}
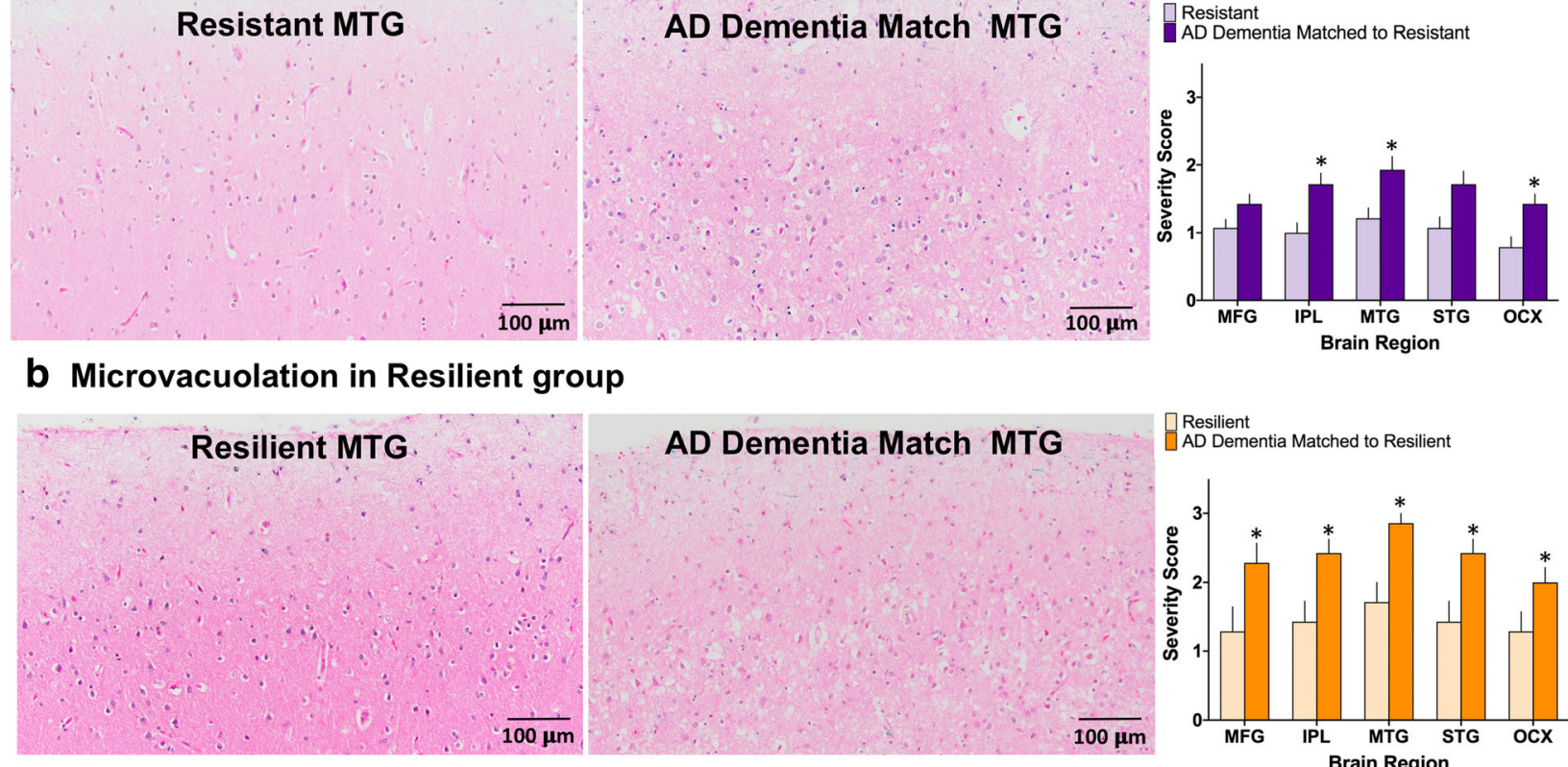

Resilient

AD Dementia Matched to Resilient

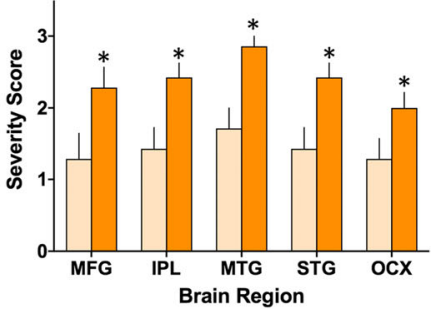

Fig. 5 Cortical microvacuolar change. The degree of neurodegenerative tissue damage was assessed in each case on H\&E/LFB-stained slides. The microvacuolar changes associated with parenchymal loss and reactive gliosis were scored on a 3-point scale in each neocortical region such that $1=$ limited to superficial layers (1-2), 2 = extends to deeper layers (3-4), and $3=$ translaminar involvement (5-6). In all AD dementia subjects, the MTG was the most severely affected. a Overall there was significantly less parenchymal damage in the resistant group in the IPL, MTG, and OC. There was a trend for less parenchymal damage in the MTG $(p=0.0588)$ and the STG $(p=0.0592)$. $\mathbf{b}$ Resilient subjects compared to AD dementia matches showed less parenchymal damage in all cortical regions assessed. (MFG, middle frontal gyrus; IPL, inferior parietal lobule; MTG, middle temporal gyrus; STG, superior temporal gyrus; and OCX, occipital cortex). ${ }^{*} p<0.05$; Wilcoxon matched-pairs signed-ranks test

\section{Perforant pathway synaptic integrity}

Loss of synapses in the perforant pathway has been shown to correlate with cognitive impairment and AD $[32,80]$. In particular, synapses within the outer molecular layer of the hippocampal dentate gyrus, which arise from layers two and three of the entorhinal cortex, are affected in AD while the neurons and synapses of the inner molecular layer remain relatively unaffected. To assess the integrity of the perforant pathway synapses we determined the relative immunointensity ratio (RIR) for synaptophysin staining of the inner and outer molecular layers of the dentate gyrus. The resilient group had a mean (SD) RIR of 0.87 (0.12), which was significantly higher than the AD dementia matched group, which had a mean (SD) of $0.73(0.06, p=0.046)$ (Fig. 8). There was no difference in mean RIR between the resistant group and their AD dementia matches (data not shown).

\section{In vivo modeling of tau and TDP-43 co-pathology}

There is a large body of literature associating pTau and pTDP-43 pathology with cognitive decline in $\mathrm{AD}[2,6$, $35,41,60,61,66,77,101]$. We confirmed these associations in this study using highly stringent selection criteria, where higher cortical pTau burden and the presence of pTDP-43 pathology were the key discriminating features between individuals who were resilient to the cognitive impact of AD pathology versus those with clinical dementia. However, it is unknown whether there is a synergistic relationship between pTau and pTDP-43 pathology. To test this, we used transgenic C. elegans expressing wild-type $4 \mathrm{R} 1 \mathrm{~N}$ human tau pan-neuronally (hTau tg) from a chromosomally integrated single, Mendelian segregating singe transgene [93]. hTau tg animals have low levels of tau protein and a very mild behavioral deficit. To test interactions between tau and TDP-43, we crossed hTau tg animals with C. elegans expressing wildtype human TDP-43 pan-neuronally (hTDP-43 tg) from a chromosomally integrated single, Mendelian segregating singe transgene [51]. hTDP-43 tg animals have low levels of TDP-43 protein and a very mild locomotion behavior dysfunction as well. To our surprise, we were unable to isolate double transgenic animals with both hTau and hTDP-43 transgenes after picking typical numbers of progeny from a cross, a situation indicative of possible synthetic lethality. To measure any synthetic lethality caused by synergistic toxicity between hTau and hTDP43 encoding transgenes, we crossed hTau tg and hTDP- 


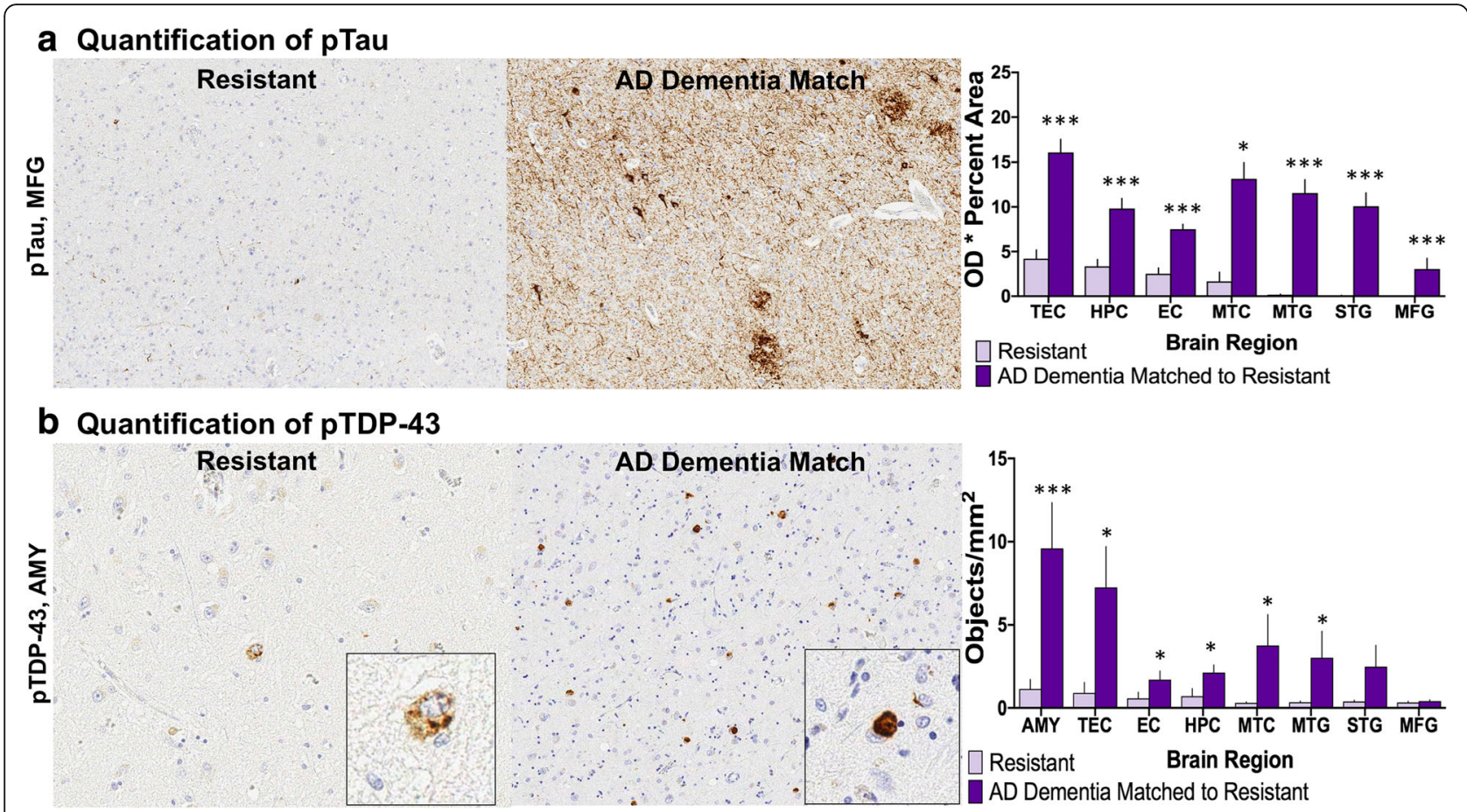

Fig. 6 Quantitative pathology for pTau and pTDP-43 in the resistant group. The resistant group had less quantitative pTau in every brain region assessed (a) and less quantitative pTDP-43 burden in the majority of brain regions assessed (b) compared to the AD dementia matches. (MFG, middle frontal gyrus; MTG, middle temporal gyrus; STG, superior temporal gyrus; MTC, mesial temporal cortex; EC, entorhinal cortex; HPC, hippocampus; TEC, transentorhinal cortex; AMY, amygdala) ${ }^{* * *} p<0.005,{ }^{*} p<0.05$; Wilcoxon matched-pairs signed-ranks test

$43 \mathrm{tg}$ animals and isolated individuals that were homozygous for the hTau tg but heterozygous for the hTDP-43 tg (hTau tg +/+; hTDP-43 tg +/-). We then scored the genotypes of the progeny from these animals for the presence of the hTDP-43 tg (Fig. 9). We found that the genotypes of progeny significantly differed from the expected Mendelian ratios for assortment of a single genetic element (Fig. 9b). As a control, we generated a similar cross between hTau tg and a transgene expressing only GFP pan-neuronally (GFP Tg). Classes of progeny produced from this cross were not significantly different from expected Mendelian ratios (Fig. 9a-b), indicating that toxicity observed is specific for individuals expressing both hTau and hTDP-43. By picking large numbers of progeny from hTau tg +/+; hTDP-43 tg +/animals, we recovered a rare class of individuals that were homozygous for both hTau and hTDP-43 transgenes (hTau tg $+/+$; hTDP-43+/+). In contrast to the hTau or hTDP tg parent animals, these individuals had severe movement dysfunction (Fig. 9c). We then tested whether protein levels of tau or TDP-43 were different in the double transgenic animals. Levels of both total and phosphorylated tau and TDP-43 were dramatically elevated in the hTau tg +/+; hTDP-43 tg +/+ double homozygotes compared to parental single transgenic animals alone (Fig. 9d). Taken together, these data indicate that tau and TDP-43 synergize in vivo exacerbating proteotoxicity.

\section{Discussion}

There is a growing body of literature describing associations with clinical and neuropathological resistance and resilience to AD neuropathologic change (ADNC), but no agreed upon standards for categorization or even nomenclature. We recognized the potential for misclassification using lenient standards for clinical dementia and ADNC and so devised a selection scheme with highly stringent criteria to define resistance (non-demented, Braak <IV, CERAD none, age 85+) and resilience (nondemented, Braak VI, CERAD frequent) to ADNC in subjects with supportive psychometric testing within two years of death. Our goal was to select for cases that would facilitate identification of pathways and pathologies for emphasis in future epidemiological, neuropathological, and translational research. Under these conditions, we identified seven individuals from the ACT study (out of 684 total autopsies) who were cognitively normal within two years of death, and yet at autopsy had severe ADNC, defined as a Braak stage of VI and a CERAD score of frequent neuritic plaques (although standardized assessment using the latest techniques resulted in reclassification of one resilient case to 


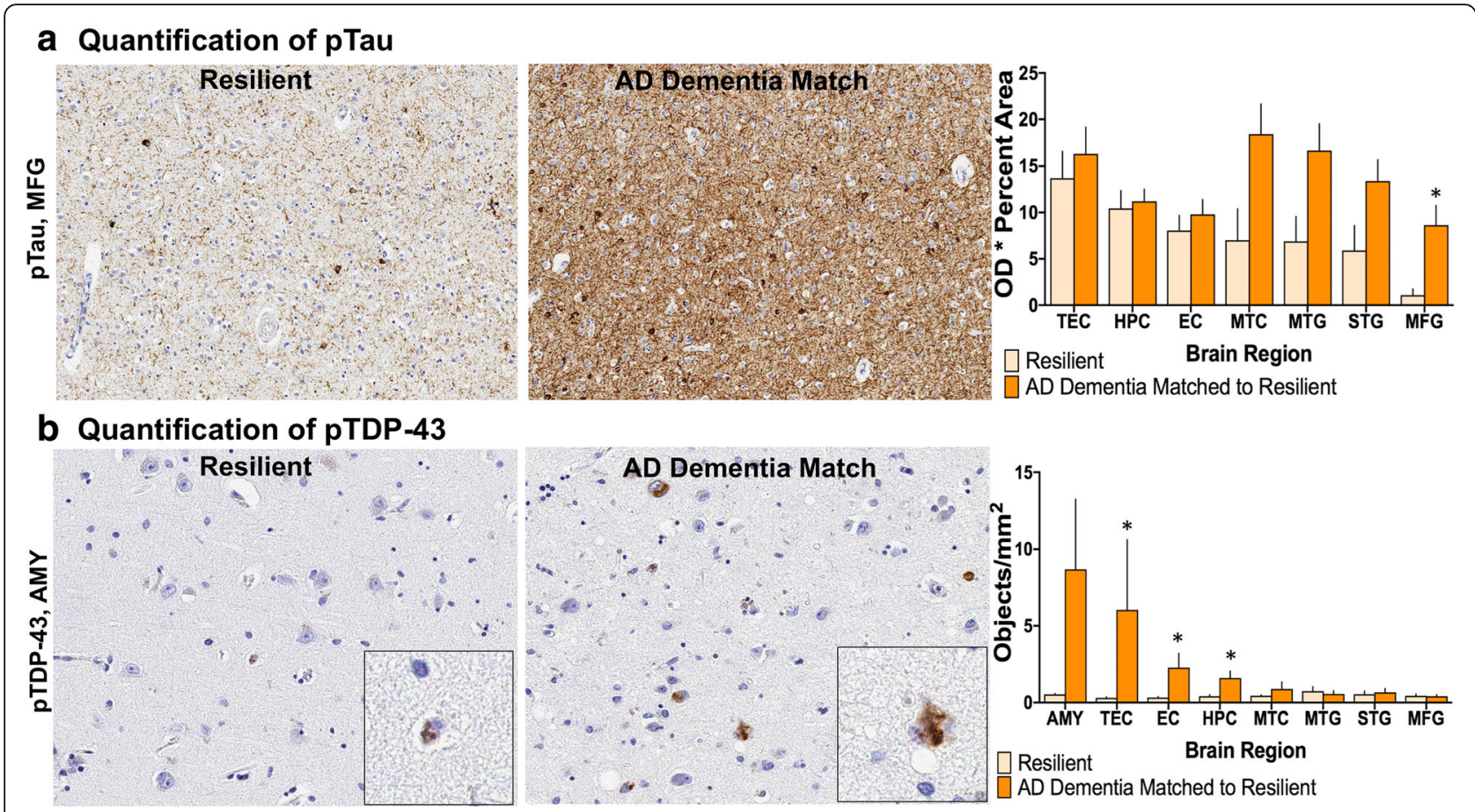

Fig. 7 Quantitative pathology for pTau and pTDP-43 in the resilient group: a The resilient group had less quantitative pTau burden in the MFG. b Quantitative assessments of pTDP-43 also revealed less pathologic burden in TEC, EC, and HPC in the resilient group compared to AD dementia matches. Amygdala showed a trend for reduced TDP-43 immunoreactivity ( $p=0.0630)$. (MFG, middle frontal gyrus; MTG, middle temporal gyrus; STG, superior temporal gyrus; MTC, mesial temporal cortex; EC, entorhinal cortex; HPC, hippocampus; TEC, transentorhinal cortex; AMY, amygdala) * $\mathrm{p}<0.05$; Wilcoxon matched-pairs signed-ranks test

Braak V). By limiting this group to those highest levels of neurofibrillary tangle distribution and neuritic plaque burden, we attempted to exclude variations in Braak stage and CERAD score as drivers of resilience. Similar considerations led us to require that the resistant group died without dementia at age $\geq 85$ and had a Braak stage of III or less and a CERAD score of none, ensuring low to no ADNC. These strict definitions created homogeneous, albeit small, groups with respect to ADNC for the purposes of identifying unique clinical characteristics that associated with each group and permitted the pathologic assessment to focus on variables beyond the current guidelines for the evaluation of ADNC.

\section{Education may play a role in resistance to developing cerebrovascular and AD pathology}

After comparing multiple participant characteristics, including years of education, smoking history, and comorbid diseases, we did not identify statistically significant differences between the resistant or resilient groups and their dementia matches. Power to detect differences across these characteristics is low in this stringentlydefined group, but there was a trend in the resistant group toward more years of education and toward reduced cerebrovascular pathology (CAA, arteriolosclerosis, and atherosclerosis). Multiple prior studies have demonstrated an inverse association between higher education and cerebrovascular pathology [21, 42, 100]; our results support these prior findings. The literature is mixed on the association between education and $\mathrm{AD}$, with some studies showing a protective effect of education on ADNC and others demonstrating lack of an association $[9,21,27,33,45,57,100]$. We did not identify an association between educational level and resilient vs. demented matches in this study, but this study may not be powered to detect small effects in this regard; further evaluation in larger samples is warranted.

\section{$A P O E \varepsilon 2$ and $\varepsilon 3$ genotypes are associated with resistance to $A D$ neuropathologic change}

We found fewer resistant cases with $\geq 1 A P O E$ \&4 allele; $\mathrm{A} \beta$ and pTDP-43 pathology have both been associated with the APOE \&4 allele [18, 52, 73, 84, 97, 102], in alignment with our findings. In contrast, resilient cases were not different from $\mathrm{AD}$ dementia matches with respect to $A P O E \& 4$ alleles. This is consistent with prior research on $\mathrm{A} \beta$ and $A P O E \varepsilon 4$, since groups in this study were matched based in part on $A \beta$ (CERAD) levels, but does not account for lower levels of pTDP-43 pathology in the resilient group. Given the rich literature associating the $A P O E \& 4$ allele with 

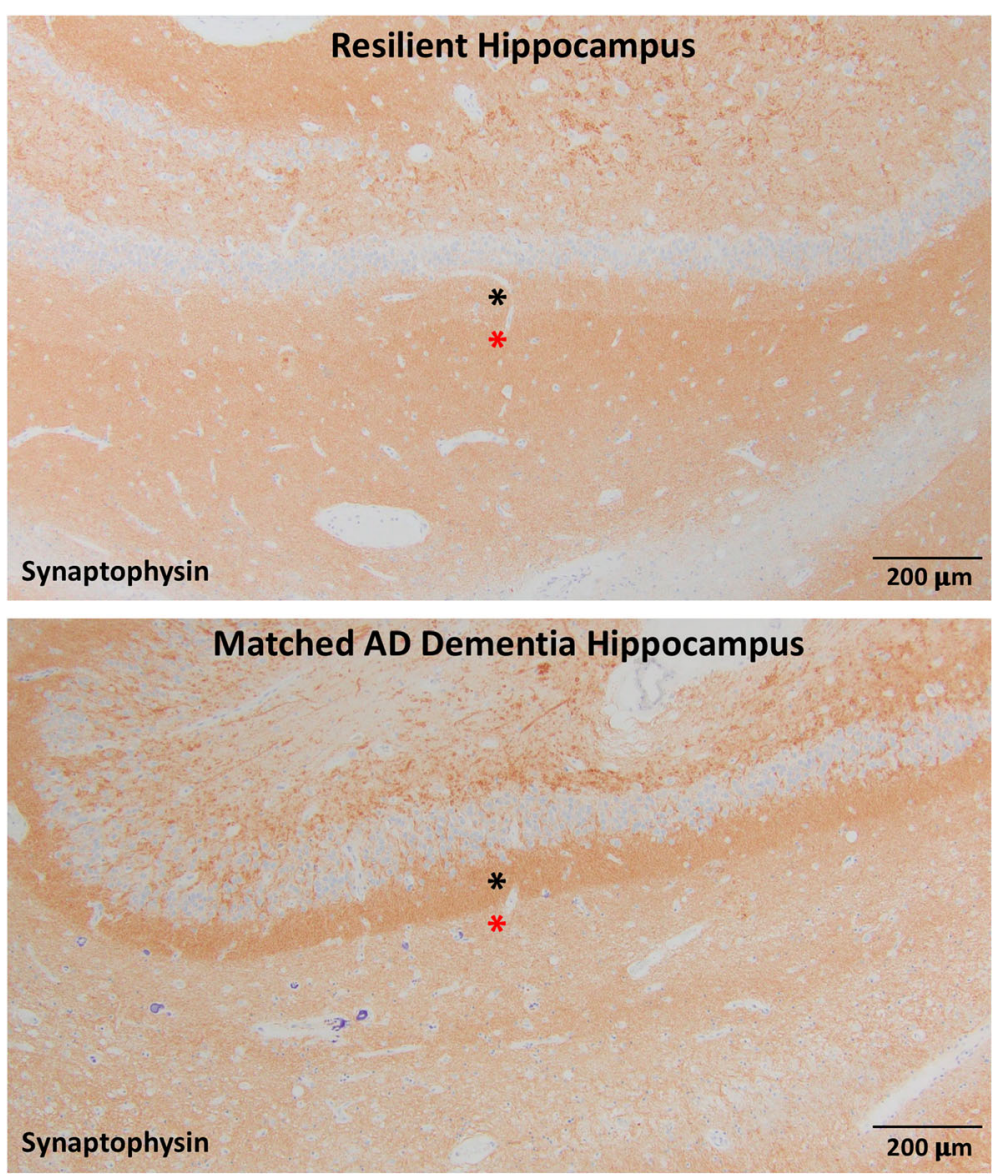

* Inner Molecular Layer

* Outer Molecular Layer

Relative Intensity Ratio :

\begin{tabular}{c}
$\begin{array}{c}\text { Intensity of outer } \\
\text { molecular layer }\end{array}$ \\
\hline $\begin{array}{c}\text { Intensity of inner } \\
\text { molecular layer }\end{array}$
\end{tabular}



Fig. 8 Synaptic integrity of the perforant pathway in the resilient group. The ratio of synaptophysin staining between the outer molecular layer of the dentate gyrus of the hippocampus (red asterisk) and the inner molecular layer (black asterisk) provides a measure of synaptic integrity of the perforant pathway. The AD dementia subjects had a reduced ratio due to a preferential reduction in staining of the outer molecular layer compared to the inner layer while the resilient group maintained a ratio closer to one, reflecting maintained synaptic health. ${ }^{*} p<0.05$, Wilcoxon matched-pairs signed-ranks test

increased risk of developing $\mathrm{AD}$, these results were not surprising. We could not control for $A P O E$ allele frequency in this study due to the small sample size, but it will be important in future larger-scale studies to control for this variable in order to better understand additional mechanisms of resistance to ADNC.

\section{Cerebrovascular pathology is lower in resilient and resistant groups}

Both resistant and resilient groups had lower rates of cerebrovascular pathology on average compared to their dementia matches, although the specific type of cerebrovascular pathology was different across 



Fig. 9 hTau and hTDP-43 synergize in vivo to drive neurotoxicity and protein accumulation. a, b Pan-neuronal expression of human TDP-43 (hTDP-43) is synthetic lethal with human Tau (hTau) in C. elegans transgenic models. Progeny from animals homozygous for hTau tg (+/+) but heterozygous for either GFP Tg or hTDP-43 tg (+/-) were picked blind and then scored for GFP Tg or hTDP-43 tg genotype. Expected Mendelian ratios for assortment of a single genetic element are 25\% (+/+), 50\% (+/-), and 25\% (-/-). Ratios of progeny from animals heterozygous for GFP $\mathrm{Tg}$ are not significantly different from Mendelian ratios ( $p=0.502$, Chi square analysis). Ratios of progeny from animals heterozygous for $h$ TDP-43 tg are significantly different from Mendelian ratios $(p<0.0001)$. $N=563$, GFP Tg. $N=561$, hTDP-43 tg. c Developmentally synchronized L4 larvae of hTau tg (+/+); TDP-43 (+/+) double homozygotes move significantly less than hTau tg (+/+) or hTDP-43 $\operatorname{tg}(+/+)$ alone $(* * * *<0.0005)$. Statistical significance was determined using one-way ANOVA with Tukey's multiple-comparison test. $\mathbf{d}$ Co-expression of hTau and hTDP-43 promotes accumulation and pathological phosphorylation of both proteins in vivo. Developmentally synchronized day 1 adult $C$. elegans were harvested and tested by immunoblot for total tau, phosphorylated tau (AT180), total TDP-43, phosphorylated TDP-43 (phospho-S409/410) and tubulin (load control). Immunoblot shown is representative of three independent replicate experiments

comparisons. Resistant cases had less CAA on average than their dementia matched group, in alignment with the low levels of parenchymal $A \beta$ found in this group. CAA has also been shown to associate with lower cognitive performance independent of the association of CAA on AD pathology [7]. Arteriolosclerosis and atherosclerosis severity was lower on average in resistant cases; interestingly these differences did not translate to fewer macro- or micro-infarcts. Macroscopic infarcts were lower in resilient cases compared with $\mathrm{AD}$ dementia matches; vascular brain injury is a common comorbid pathology in late onset $\mathrm{AD}[17,25,49,85,91,99]$ and macroscopic infarcts have been shown to contribute to cognitive decline and dementia in other cohorts [86]. Thus, one component of the resilience phenotype may include resistance to macroscopic infarcts in the face of severe ADNC, but this effect alone does not entirely explain the resilience phenotype in this group.

\section{Extent of amyloid pathology may be lower in resilient} individuals

There is a debate as to whether assessing the extent of $A \beta$ pathology (Thal phase) in addition to the cortical burden of neuritic plaques (CERAD score) in the evaluation of ADNC provides useful information with respect to ADNC-related neurodegeneration and dementia [48, 74, 87]. For historical reasons, Thal phase was not available as a selection criterion and therefore was prospectively evaluated in every case for this study. Although overall cortical $A \beta$ burden was similar in resilient cases and $\mathrm{AD}$ dementia matches, there was greater $\mathrm{A} \beta$ plaque burden in regions of higher Thal phases (brainstem, Thal phase 4 and cerebellum, Thal phase 5) in the AD dementia matches, and only two resilient cases had any cerebellar $A \beta$ burden. These results, albeit from a small and highly selected group, support a role for assessment of $\mathrm{A} \beta$ distribution in consideration of ADNC to classify cases with respect to dementia to more accurately assess 
overall ADNC burden. Moreover, this information is critical in determining the impact of $A \beta$ extent and burden in relation to ADNC phenotypic penetrance and, specifically, the functional impacts of $A \beta$ involvement of brainstem and cerebellum. In particular the cerebellum is increasingly recognized for its role in cognition and therefore understanding cerebellar sparing of amyloid pathology may prove to be particularly important in elucidating mechanisms of resilience $[34,83]$.

\section{Despite using Braak stage for selection, cortical pTau is significantly lower in resilient cases}

We applied stringent ADNC selection criteria to limit the potential for misclassification of cases as resilient due to lower pathologic burden, but even with these criteria, our quantitative algorithms to assess ADNC using digitized slides of immunohistochemically-stained tissue sections still stratified demented and non-demented cases by pathology. This highlights the limitations of current diagnostic guidelines to precisely classify pathologic burden. While several studies have used some form of quantitative or semi-quantitative assessment of pTau burden $[58,68,79]$, this approach allowed us to obtain a quantitative measure of proteins of interest from readily available tissue sections that have also been reviewed by standard neuropathologic techniques. Using this technique to measure pTau, we found that resilient cases had lower overall levels of pTau in the middle frontal gyrus on average compared to the matched AD dementia group, supporting the notion that quantitative pTau burden, which includes pTau-positive neurites in addition to neurofibrillary tangles, may predict cognitive decline better than Braak stage. While prior studies suggest this through semi-quantitative assessments of pTau pathology $[1,64,65]$, ours is the first study to quantitatively demonstrate lower levels of pTau in the same sections that underwent standard Braak staging and further argues for consideration of both extent and burden of pathologic proteins in $\mathrm{AD}$.

\section{Limbic-predominant age-related TDP-43 encephalopathy neuropathologic change (LATE-NC) occurs more often in the context of $A D$ dementia than in resilient or resistant groups}

We hypothesized that individuals who are either resilient or resistant to ADNC would have less co-morbid pathologies. In addition to assessment of vascular brain injury (described previously), we performed a thorough assessment for AD-associated pathologic proteins ( $\alpha$-synuclein and pTDP-43). While we found no significant differences in Lewy body distribution or $\alpha$-synuclein pathology between groups with and without dementia, the presence of pTDP-43 pathology was strikingly specific for the AD dementia subjects. Only $2 / 14$ resistant cases had pTDP-43 pathology (compared with 13/14 AD dementia matches) and 1/7 resilient cases exhibited some degree of pTDP-43 pathology (compared with 6/7 $\mathrm{AD}$ dementia matches). TDP-43, first identified as the major pathologic protein in sporadic amyotrophic lateral sclerosis (ALS) and the majority of frontotemporal lobar degeneration (FTLD) cases, has only more recently been described in association with AD. Several studies report a high prevalence of pTDP-43 in late-onset AD, reaching up to $50 \%$ in some cohorts $[2,30,60,96]$. The pathologic distribution of pTDP-43 in AD is distinct from that seen in ALS/FTLD, where the neocortex is prominently involved. In AD, pTDP-43 pathology overlaps with that of pTau, demonstrating a predilection for the amygdala and hippocampus and later involving neocortex $[4,38$, 39, 59]. Further, neuroimaging-neuropathologic correlations have demonstrated a relationship between pTDP43 pathology at autopsy and more rapid progression of hippocampal atrophy on antemortem MRI $[36,40]$. Individuals who are found at autopsy to have pTDP-43 aggregates are more likely to have been cognitively impaired, as seen in this study, and others have also shown an association between dementia and pTDP-43 pathology in the setting of significant ADNC [40, 41, 60]. Indeed, this age-related pattern of pTDP- 43 pathology has recently been considered through a consensus processes organized by NIA, where the term Limbicpredominant, Age-related, TDP-43 Encephalopathy Neuropathologic Change (LATE-NC) has been assigned to describe the neuropathology of age-related pTDP-43 proteinopathy [66]. In the consensus paper, the authors point out that LATE-NC is often, but not always, associated with ADNC and the relationships between LATE and $\mathrm{AD}$ remain incompletely characterized. In this study, the pTDP-43 pathologic findings demonstrate increased frequency of LATE-NC in dementia compared with resistant and resilient cases.

Given the paucity of $A \beta$ pathology in the setting of mild tauopathy, the resistant cases are considered to have either definite (Thal phase $0, n=5$ ) or possible (Thal phase $1, n=9$ ) primary age-related tauopathy (PART) [20]. There is limited literature regarding pTDP43 pathology in the context of PART but the incidence in our study $(14 \%)$ is lower than that reported by Josephs et al. [37] in which approximately $29 \%$ of PART cases were found to have very low stage of TDP-43 pathology, the majority of which were limited to the amygdala (LATE-NC stage 1). In our study, one resistant subject had LATE-NC stage 1 (amygdala pTDP-43 pathology) while the other had LATE-NC stage 2 (hippocampal pTDP-43 pathology). Both cases had burdensome pTDP-43 deposits, unlike the majority of cases described by Josephs et al., in which often only a single inclusion was identified [37]. Therefore, the lower percentage of 
pTDP-43 positive cases in this group may in part be due to sampling bias. Regardless, similar to this prior study, we also find no association between pTDP-43 pathology and cognitive status in the resistant group.

The findings in the resilient group are consistent with other reports associating cognitive decline in late-onset $\mathrm{AD}$ and the presence of pTDP-43 pathology [2, 26, 35, 40, 60, 66, 97, 101]. To illustrate this association, we highlight two contrasting cases from the resilient cohort, subject 7 and subject 4 (see Fig. 1). Subject 7 is a prime example of resilience, with a high, stable CASI score (94/100 at both the penultimate and final examinations) and significant quantitative pTau pathology in the middle frontal cortex on par with that seen in the matched AD dementia subjects (5.11 compared to $8.63 \pm 2.13$ ). Notably, however, this subject lacks pTDP-43 pathology. Therefore, while comparable to the AD dementia group with respect to standard and quantitative assessment of $\mathrm{ADNC}$, a striking discriminating feature in this case is the lack of LATE-NC. Conversely, Subject 4 has slightly lower levels of pTau burden in the middle frontal gyrus compared to the matched AD dementia group (1.22 compared to $8.63 \pm 2.13$ ), but did have LATE-NC and a decline in CASI from the penultimate (98) to the final (88) examinations. This individual did not meet criteria for a diagnosis of dementia but did demonstrate a decline in their cognitive function in the setting of combined ADNC and LATE-NC. Given the limited nature of the groups, these findings cannot be used to generate broad conclusions about causation or extrapolate to the larger cohort, however they do suggest an association between LATE-NC and cognitive decline in the setting of ADNC.

Of note, while we did not specifically assess for the neuropathologic subtype of AD (typical, limbicpredominant, and hippocampal sparing) as described by Murray et al. [58], based on the quantitative pTau data, the vast majority of the cases would likely be considered limbic-predominant given the increased optical density of pTau staining in hippocampus compared to the middle frontal gyrus (24 out of 28 cases with severe ADNC had a hippocampus/MFG tau optical density ratio of > 1.5). The remaining four cases had more typical $A D$ pathology with slightly greater optical density pTau staining in MFG than hippocampus, and there were no cases of hippocampal-sparing ADNC. These data suggest that the association between pTau and pTDP-43 is important in the limbic-predominant subtype but given the small number, associations between pTau and pTDP-43 cannot be assessed in other subtypes of ADNC in this study. Although limited, the available literature on this topic has shown that associations between cognitive decline and pTDP-43 are strong in the limbicpredominant subtype of $\mathrm{AD}$ but are lacking in the other subtypes [81]. As a single case in point, there was one subject in the AD dementia group matched to the resistance group who had typical AD pathology instead of limbic-predominant, and this was the one subject that lacked pTDP-43 pathology in this cohort. Additional studies are warranted to determine whether distinct pathways are at play for the different pathologic subtypes of ADNC and if the role of pTDP-43 is of particular importance for limbic-predominant ADNC.

We also note the segregation of vascular brain injury (VBI) pathology in AD dementia subjects compared with resilient and resistant groups, which may suggest a relationship between VBI and pTDP43. Thus, the near complete segregation of LATE-NC with dementia strongly supports the notion that resilience to ADNC is in part due to resistance to PTDP-43 pathology, and this may be related to mechanisms of VBI as has been previously postulated, particularly with respect to pTDP-43 and hippocampal sclerosis [2, 69-71]. Future studies modeling this interaction are needed to further understand the association.

\section{Both resistant and resilient groups demonstrate lack of tissue damage on routine histologic evaluation}

In an attempt to compare ADNC with cerebral cortical injury, we evaluated cerebral cortex neurodegeneration from H\&E/LFB-stained sections adjacent to those stained for pathologic peptides by assessing the degree of microvacuolation of the cortex, believed to represent parenchymal loss and associated reactive gliosis. In both resistant and resilient groups there was less tissue damage compared to their matched $\mathrm{AD}$ dementia groups. This discrepancy was most prominent in temporal cortex. Given the lack of pathology present in the resistant group this finding was not surprising. Many people in the resilient group had substantial $A \beta$ pathology in cortical regions without evidence of prominent microvacuolation, but as a group showed reduced levels of pTau burden and a lack of pTDP-43 pathology. This data, in combination with similar findings (with respect to pTDP-43 and pTau) in the resistant group, suggests that these pathologic proteins may be specifically associated and perhaps interact to promote neurodegenerative tissue changes. Because this is a cross-sectional autopsy study, we cannot determine whether the association between microvacuolation and pTau and pTDP-43 pathology is a cause, consequence, or coincidence.

\section{Synaptic integrity of the perforant pathway is maintained} in resilient individuals relative to those with $A D$ dementia The hippocampus is involved in the earliest stages of supratentorial pTau pathology in the progression of $\mathrm{AD}$, and is an early site of pTDP-43 pathology that correlates with cognitive symptoms [35, 36, 56]. Hippocampal 
perforant pathway synapse loss is believed to be an early change in the progression of $\mathrm{AD}$ so we set out to compare perforant pathway synaptic injury between resilient/resistant groups and AD dementia matches following an approach previously published by Robinson et al. [71]. We found significantly increased synaptic staining in the outer molecular layer of the hippocampus of participants in the resilient group compared to the $\mathrm{AD}$ dementia matched group, but this relationship was not apparent in comparing resistant cases and matched $\mathrm{AD}$ dementia subjects. Thus, while tempting to attribute increased synaptic staining to preserved synaptic integrity as a possible basis for the resilience phenotype, this relationship is likely to be more complicated. Given the small sample size, follow-up assessment of the larger ACT cohort is necessary, as well as experimental studies, in order to better understand these pathways and determine specific interactions between pathologic peptides and neuronal and synaptic function and toxicity.

\section{TDP-43 and tau may interact synergistically to increase the risk of dementia in elderly individuals}

Studies in stringently selected human cohorts of resistance and resilience and carefully matched demented subjects with severe ADNC revealed significant differences in cortical pTau burden and the presence of pTDP-43 pathology (LATE-NC). Hyperphosphorylated tau has long been described as a diagnostic pathologic feature of $\mathrm{AD}$, and its distribution throughout the brain (Braak stage) correlates well with cognitive impairment $[29,64,67]$. However, using quantitative methods, we show that despite having the same Braak stage as their matched AD dementia group, the resilient group had less pTau burden on average in cortical regions. The resilient group also tended to lack LATE-NC, and although the mechanism is currently unknown, this study and others suggest that pTDP-43 together with pTau is associated with increased risk of dementia in elderly people [18]. While other studies have shown that the incidence of concomitant pTDP-43 pathology increases with increased Braak stage [35, 36, 38, 88], this study is the first to use quantitative methods to show that higher pTau burden is associated with LATE-NC. Although this is an intriguing relationship, the underlying mechanism cannot be further elucidated in autopsy tissue. We therefore developed a model in C. elegans to test whether there may be more than a summative association between TDP-43 and tau. In C. elegans, we found that coexpressing human (h) Tau and hTDP-43 in the same animal markedly exacerbates the phenotypes over those caused by either peptide in isolation, including synthetic lethality and severe movement dysfunction, and dramatically increased hTau and hTDP-43 protein accumulation. The combined phenotypes were greater than what would be expected if the interaction were simply additive, suggesting a toxic synergism between hTau and hTDP-43. Biochemical assays show that there is more pathological phosphorylated hTDP-43 and hTau present in worms with combined expression than when either protein is expressed alone, further supporting synergistic toxicity between the two peptides that might ultimately explain the strong association of pTDP-43 pathology and dementia with respect to both resistance and resilience. Future studies using this novel tool to study interactions between hTDP-43 and hTau in vivo, and development of vertebrate animal and human cell culture experimental systems, will be critical to better understanding mechanisms of neurodegeneration that exceed resistance and resilience mechanisms resulting in ADNC-related dementia.

\section{Limitations}

By design, dementia-free ACT participants report diseases and health behaviors more proximal to death than demented participants. No difference was detected in Charlson Comorbidity Index between the resistant group and their AD dementia matches. This suggests the resistant group might have maintained better health into older age compared to the demented subjects, since their Charlson Index less than two years before death was similar to the demented group nearly seven years before death. Future studies could be designed to compare these differences directly.

\section{Conclusions}

These results should be considered preliminary; the groups were stringently selected to be informative but as a result were quite small. However, this study is the first to extensively characterize two cognitively intact groups, specifically defined as resilient or resistant based on the presence or absence of severe ADNC and the first to describe LATE-NC in the context of resistance and resilience to ADNC. It is also the first to model combined hTau and hTDP-43 in C. elegans and demonstrate the synergistic proteotoxic effects. Because the study utilizes a highly selected cohort of ADNC outliers, additional work must extend these neuropathologic approaches to larger longitudinal cohort studies, including the entirety of the ACT autopsy cohort, as well as address the underlying pathways of tau and TDP-43 synergism in the context of late-onset $\mathrm{AD}$ to elucidate novel pathways for the exploration of treatment targets for AD.

\footnotetext{
Abbreviations

ACT: Adult Changes in Thought; AD: Alzheimer's Disease; ADNC: Alzheimer's Disease Neuropathologic Change; ADRC: Alzheimer's Disease Research Center; ADRD: Alzheimer's Disease and Related Dementias; ALS: amyotrophic lateral sclerosis; $A \beta$ : amyloid $\beta$; CAA: cerebral amyloid angiopathy; CASI: Cognitive Abilities Screening Instrument; CERAD: Consortium to Establish a Registry for Alzheimer's Disease; FFPE: Formalin fixed paraffin
} 
embedded; FTLD: Frontotemporal lobar degeneration; GH: Group Health; H\&E: Hematoxylin and eosin; IHC: Immunohistochemistry; KPW: Kaiser Permanente Washington; LATE: Limbic-predominant age-related TDP-43 encephalopathy; LATE-NC: Limbic-predominant age-related TDP-43 encephalopathy neuropathologic change; LBD: Lewy Body disease; LFB: Luxol fast blue; NFT: Neurofibrillary tangle; NIA-AA: National Institute on AgingAlzheimer's Association; OD: Optical density; RIR: Relative intensity ratio; ROI: Region of interest; SD: Standard deviation; TDP-43: Transactive response DNA binding protein $43 \mathrm{kDa}$; UW: University of Washington; VBI: Vascular brain injury; $\mu \mathrm{VB}$ I: Microvascular brain injury

\section{Acknowledgements}

We thank Kim Howard and Lisa Keene for outstanding technical assistance and Allison Beller for administrative support. For image analysis services we thank the NW BioTrust, a core service for patient consenting, and NWBioSpecimen, a core service for procurement and annotation of research biospecimens supported by National Cancer Institute grant P30 CA015704 (G. Gilliland, principal investigator [PI]), Institute of Translational Health Sciences grant UL1 TR000423 (M. Disis, PI), the University of Washington School of Medicine and Department of Pathology, and Fred Hutchinson Cancer Research Center. We thank the Developmental Studies Hybridoma Bank (NICHD) for the $\beta$-tubulin primary antibody E7.

\section{Authors' contributions}

CSL performed the neuropathologic examination for all cases, analyzed and interpreted the neuropathologic data, and was a major contributor in writing the manuscript. BTB analyzed and interpreted the clinical data and was a major contributor to the writing the manuscript. NFL designed, analyzed and interpreted the $C$. elegans experiments and results and was a major contributor in writing the manuscript. HNC performed the C. elegans experiments. MDK performed data acquisition and analysis on digitallyscanned slides. LEG performed statistics on the neuropathologic data. JH designed algorithms for and performed data acquisition on the digitallyscanned immunohistochemically stained slides, as well as contributed to the writing of these methods. MD, KD-R, SJ, TJG, PKC, and EBL were involved in study design and concept and provided expertise in their respective areas. BCK designed and interpreted the C. elegans experiments and data and was involved in study design and concept. TDB and CDK conceptualized the study design and provided expertise in the evaluation, analysis, and interpretation of the data. All authors read and approved the final manuscript.

\section{Funding}

This work was supported by grants from the National Institutes of Health [P50 AG005136 for the UW Alzheimer's Disease Research Center; U01 AG006781 for the Adult Changes in Thought study; RF1 AG055474 and R01 NS064131 to B.C.K] and the Department of Veterans Affairs [Merit Review Grants \#101BX002619 to B.C.K. and \#101BX004044 to N.F.L.] and the Nancy and Buster Alvord Endowment [to C.D.K].

\section{Availability of data and materials}

The datasets used and/or analysed during the current study are available from the corresponding author on reasonable request.

\section{Ethics approval and consent to participate}

This study was approved by the Group Health (GH)/Kaiser Permanente Washington (KPW) and University of Washington (UW) Human Subjects Review Committees.

\section{Consent for publication}

Not applicable.

\section{Competing interests}

The authors declare that they have no competing interests.

\section{Author details}

'Division of Neuropathology, Department of Pathology, University of Washington, Seattle, WA 98104, USA. ${ }^{2}$ Kaiser Permanente Washington Health Research Institute, Seattle, WA, USA. ${ }^{3}$ Geriatrics Research Education and Clinical Center, Veterans Affairs Puget Sound Health Care System, Seattle, WA, USA. ${ }^{4}$ Division of Gerontology and Geriatric Medicine, Department of
Medicine, University of Washington, Seattle, WA, USA. ${ }^{5}$ Department of Medicine, University of Washington, Seattle, WA, USA. 'Department of Neurology, University of Washington, Seattle, Washington, USA. 'Deparment of Radiology, University of Washington, Seattle, Washington, USA. ${ }^{8}$ Department of Psychiatry and Behavioral Sciences, University of Washington, Seattle, Washington, USA. 'Division of Medical Genetics, Department of Medicine, University of Washington, Seattle, WA, USA.

Received: 15 May 2019 Accepted: 16 May 2019

Published online: 07 June 2019

\section{References}

1. Abner EL, Kryscio RJ, Schmitt FA, Santacruz KS, Jicha GA, Lin Y, Neltner JM, Smith CD, Van Eldik LJ, Nelson PT (2011) "End-stage" neurofibrillary tangle pathology in preclinical Alzheimer's disease: fact or fiction? J Alzheimers Dis 25:445-453. https://doi.org/10.3233/JAD-2011-101980

2. Amador-Ortiz C, Lin W-L, Ahmed Z, Personett D, Davies P, Duara R, GraffRadford NR, Hutton ML, Dickson DW (2007) TDP-43 immunoreactivity in hippocampal sclerosis and Alzheimer's disease. Ann Neurol 61:435-445, https://doi.org/10.1002/ana.21154

3. American Psychiatric Association. Task force on DSM-IV. (2000) Diagnostic and statistical manual of mental disorders: DSM-II. Am Psychiatr Publ. doi: http://dx.doi.org.lrc1.usuhs.edu/10.1176/appi.books.9780890425596

4. Aoki N, Murray ME, Ogaki K, Fujioka S, Rutherford NJ, Rademakers R, Ross OA, Dickson DW (2015) Hippocampal sclerosis in Lewy body disease is a TDP-43 proteinopathy similar to FTLD-TDP type a. Acta Neuropathol. https://doi.org/10.1007/s00401-014-1358-z

5. Arenaza-Urquijo EM, Vemuri P (2018) Resistance vs resilience to Alzheimer disease: clarifying terminology for preclinical studies. Neurology 90:695-703. https://doi.org/10.1212/WNL.0000000000005303

6. Arriagada PV, Growdon JH, Hedley-Whyte ET, Hyman BT (1992) Neurofibrillary tangles but not senile plaques parallel duration and severity of Alzheimer's disease. Neurology. https://doi.org/10.1038/186476a0

7. Arvanitakis Z, Leurgans SE, Wang Z, Wilson RS, Bennett DA, Schneider JA (2011) Cerebral amyloid angiopathy pathology and cognitive domains in older persons. Ann Neurol 69:320-327. https://doi.org/10.1002/ana.22112

8. Bennett DA, Schneider JA, Arvanitakis Z, Kelly JF, Aggarwal NT, Shah RC, Wilson RS (2006) Neuropathology of older persons without cognitive impairment from two community-based studies. Neurology 66:1837-1844. https://doi.org/10.1212/01.wnl.0000219668.47116.e6

9. Bennett DA, Wilson RS, Schneider JA, Evans DA, Mendes de Leon CF, Arnold SE, Barnes LL, Bienias JL (2003) Education modifies the relation of AD pathology to level of cognitive function in older persons. Neurology 60 : 1909-1915

10. Borgatti A, Koopmeiners JS, Sarver AL, Winter AL, Stuebner K, Todhunter D, Rizzardi AE, Henriksen JC, Schmechel S, Forster CL, Kim J-H, Froelich J, Walz J, Henson MS, Breen M, Lindblad-Toh K, Oh F, Pilbeam K, Modiano JF, Vallera DA (2017) Safe and effective sarcoma therapy through bispecific targeting of EGFR and UPAR. Mol Cancer Ther 16:956-965. https://doi.org/ 10.1158/1535-7163.MCT-16-0637

11. Braak H, Alafuzoff I, Arzberger T, Kretzschmar H, Tredici K (2006) Staging of Alzheimer disease-associated neurofibrillary pathology using paraffin sections and immunocytochemistry. Acta Neuropathol. https://doi.org/10. 1007/s00401-006-0127-z

12. Braak H, Braak E (1991) Neuropathological stageing of Alzheimer-related changes. Acta Neuropathol

13. Brenner S (1974) The genetics of Caenorhabditis elegans. Genetics. https:// doi.org/10.1002/cbic.200300625

14. Buchman AS, Yu L, Wilson RS, Lim A, Dawe RJ, Gaiteri C, Leurgans SE, Schneider JA, Bennett DA (2019) Physical activity, common brain pathologies, and cognition in community-dwelling older adults. Neurology. https://doi.org/10.1212/WNL.0000000000006954

15. Chan D, Shafto M, Kievit R, Matthews F, Spink M, Valenzuela M, Henson RN (2018) Lifestyle activities in mid-life contribute to cognitive reserve in latelife, independent of education, occupation, and late-life activities. Neurobiol Aging 70:180-183. https://doi.org/10.1016/j.neurobiolaging.2018.06.012

16. Charlson ME, Pompei P, Ales KL, MacKenzie CR (1987) A new method of classifying prognostic comorbidity in longitudinal studies: development and validation. J Chronic Dis 40:373-383

17. Cholerton B, Larson EB, Baker LD, Craft S, Crane PK, Millard SP, Sonnen JA, Montine TJ (2013) Neuropathologic correlates of cognition in a population- 
based sample. J Alzheimers Dis 36:699-709. https://doi.org/10.3233/JAD130281

18. Chornenkyy Y, Fardo DW, Nelson PT (2019) Tau and TDP-43 proteinopathies: kindred pathologic cascades and genetic pleiotropy. Lab Investig. https:// doi.org/10.1038/s41374-019-0196-y

19. Corrada MM, Sonnen JA, Kim RC, Kawas CH (2016) Microinfarcts are common and strongly related to dementia in the oldest-old: the 90+ study. Alzheimers Dement 12:900-908. https://doi.org/10.1016/j.jalz.2016.04.006

20. Crary JF, Trojanowski JQ, Schneider JA, Abisambra JF, Abner EL, Alafuzoff I, Arnold SE, Attems J, Beach TG, Bigio EH, Cairns NJ, Dickson DW, Gearing M, Grinberg LT, Hof PR, Hyman BT, Jellinger K, Jicha GA, Kovacs GG, Knopman DS, Kofler J, Kukull WA, Mackenzie IR, Masliah E, McKee A, Montine TJ, Murray ME, Neltner JH, Santa-Maria I, Seeley WW, Serrano-Pozo A, Shelanski ML, Stein T, Takao M, Thal DR, Toledo JB, Troncoso JC, Vonsattel JP, White CL 3rd, Wisniewski T, Woltjer RL, Yamada M, Nelson PT (2014) Primary agerelated tauopathy (PART): a common pathology associated with human aging. Acta Neuropathol 128:755-766. https://doi.org/10.1007/s00401-0141349-0

21. Del Ser T, Hachinski V, Merskey H, Munoz DG (1999) An autopsy-verified study of the effect of education on degenerative dementia. Brain 122 ( Pt(1):2309-2319

22. Dickson DW, Braak H, Duda JE, Duyckaerts C, Gasser T, Halliday GM, Hardy J, Leverenz JB, Del Tredici K, Wszolek ZK, Litvan I (2009) Neuropathological assessment of Parkinson's disease: refining the diagnostic criteria. Lancet Neurol 8:1150-1157. https://doi.org/10.1016/S1474-4422(09)70238-8

23. Dowling NM, Tomaszewski Farias S, Reed BR, Sonnen JA, Strauss ME, Schneider JA, Bennett DA, Mungas D (2011) Neuropathological associates of multiple cognitive functions in two community-based cohorts of older adults. J Int Neuropsychol Soc. https://doi.org/10.1017/S1355617710001426

24. Engelman M, Agree EM, Meoni LA, Klag MJ (2010) Propositional density and cognitive function in later life: findings from the precursors study. J Gerontol B Psychol Sci Soc Sci 65:706-711. https://doi.org/10.1093/geronb/ gbq064

25. Flanagan M, Larson EB, Latimer CS, Cholerton B, Crane PK, Montine KS, White LR, Keene CD, Montine TJ (2016) Clinical-pathologic correlations in vascular cognitive impairment and dementia. Biochim Biophys Acta - Mol Basis Dis. https://doi.org/10.1016/j.bbadis.2015.08.019

26. Flanagan ME, Cholerton B, Latimer CS, Hemmy LS, Edland SD, Montine KS, White LR, Montine TJ (2018) TDP-43 Neuropathologic associations in the Nun study and the Honolulu-Asia aging study. J Alzheimers Dis. https://doi. org/10.3233/JAD-180162

27. Fritsch T, McClendon MJ, Smyth KA, Lerner AJ, Chen CH, Petot GJ, Debanne SM, Soas A, Friedland RP (2001) Effects of educational attainment on the clinical expression of Alzheimer's disease: results from a research registry Am J Alzheimers Dis Other Dement 16:369-376. https://doi.org/10.1177/ 153331750101600606

28. Gow AJ, Pattie A, Deary IJ (2017) Lifecourse activity participation from early, mid, and later adulthood as determinants of cognitive aging: the Lothian birth cohort 1921. J Gerontol B Psychol Sci Soc Sci 72:25-37. https://doi.org/ 10.1093/geronb/gbw124

29. Grober E, Dickson D, Sliwinski MJ, Buschke H, Katz M, Crystal H, Lipton RB (1999) Memory and mental status correlates of modified Braak staging. Neurobiol Aging 20:573-579

30. Higashi S, Iseki E, Yamamoto R, Minegishi M, Hino H, Fujisawa K, Togo T, Katsuse O, Uchikado H, Furukawa Y, Kosaka K, Arai H (2007) Concurrence of TDP-43, tau and a-synuclein pathology in brains of Alzheimer's disease and dementia with Lewy bodies. Brain Res. https://doi.org/10.1016/..brainres. 2007.09.048

31. Hyman BT, Phelps CH, Beach TG, Bigio EH, Cairns NJ, Carrillo MC, Dickson DW, Duyckaerts C, Frosch MP, Masliah E, Mirra SS, Nelson PT, Schneider JA Thal DR, Thies B, Trojanowski JQ, Vinters HV, Montine TJ (2012) National Institute on Aging-Alzheimer's association guidelines for the neuropathologic assessment of Alzheimer's disease. Alzheimers Dement. https://doi.org/10.1016/j.jalz.2011.10.007

32. Hyman BT, Van Hoesen GW, Kromer LJ, Damasio AR (1986) Perforant pathway changes and the memory impairment of Alzheimer's disease. Ann Neurol. https://doi.org/10.1002/ana.410200406

33. lacono D, Zandi P, Gross M, Markesbery WR, Pletnikova O, Rudow G, Troncoso JC (2015) APOepsilon2 and education in cognitively normal older subjects with high levels of AD pathology at autopsy: findings from the Nun study. Oncotarget 6:14082-14091. https:/doi.org/10.18632/oncotarget.4118
34. Jacobs HIL, Hopkins DA, Mayrhofer HC, Bruner E, van Leeuwen FW, Raaijmakers W, Schmahmann JD (2018) The cerebellum in Alzheimer's disease: evaluating its role in cognitive decline. Brain 141:37-47. https://doi. org/10.1093/brain/awx194

35. James BD, Wilson RS, Boyle PA, Trojanowski JQ, Bennett DA, Schneider JA (2016) TDP-43 stage, mixed pathologies, and clinical Alzheimer's-type dementia. Brain 139:2983-2993. https://doi.org/10.1093/brain/aww224

36. Josephs KA, Dickson DW, Tosakulwong N, Weigand SD, Murray ME, Petrucelli L, Liesinger AM, Senjem ML, Spychalla AJ, Knopman DS, Parisi JE, Petersen RC, Jack CRJ, Whitwell JL (2017) Rates of hippocampal atrophy and presence of post-mortem TDP-43 in patients with Alzheimer's disease: a longitudinal retrospective study. Lancet Neurol 16:917-924. https://doi.org/ 10.1016/S1474-4422(17)30284-3

37. Josephs KA, Murray ME, Tosakulwong N, Whitwell JL, Knopman DS, Machulda MM, Weigand SD, Boeve BF, Kantarci K, Petrucelli L, Lowe VJ, Jack CRJ, Petersen RC, Parisi JE, Dickson DW (2017) Tau aggregation influences cognition and hippocampal atrophy in the absence of betaamyloid: a clinico-imaging-pathological study of primary age-related tauopathy (PART). Acta Neuropathol 133:705-715. https://doi.org/10. 1007/s00401-017-1681-2

38. Josephs KA, Murray ME, Whitwell JL, Parisi JE, Petrucelli L, Jack CR, Petersen RC, Dickson DW (2014) Staging TDP-43 pathology in Alzheimer's disease. Acta Neuropathol 127:441-450. https://doi.org/10.1007/s00401-013-1211-9

39. Josephs KA, Murray ME, Whitwell JL, Tosakulwong N, Weigand SD, Petrucelli L, Liesinger AM, Petersen RC, Parisi JE, Dickson DW (2016) Updated TDP-43 in Alzheimer's disease staging scheme. Acta Neuropathol 131:571-585. https://doi.org/10.1007/s00401-016-1537-1

40. Josephs KA, Whitwell JL, Knopman DS, Hu WT, Stroh DA, Baker M, Rademakers R, Boeve BF, Parisi JE, Smith GE, Ivnik RJ, Petersen RC, Jack CRJ, Dickson DW (2008) Abnormal TDP-43 immunoreactivity in AD modifies clinicopathologic and radiologic phenotype. Neurology 70:1850-1857. https://doi.org/10.1212/01.wnl.0000304041.09418.b1

41. Josephs KA, Whitwell JL, Weigand SD, Murray ME, Tosakulwong N, Liesinger AM, Petrucelli L, Senjem ML, Knopman DS, Boeve BF, Ivnik RJ, Smith GE, Jack CRJ, Parisi JE, Petersen RC, Dickson DW (2014) TDP-43 is a key player in the clinical features associated with Alzheimer's disease. Acta Neuropathol 127:811-824. https://doi.org/10.1007/s00401-014-1269-z

42. Jung N-Y, Cho H, Kim YJ, Kim HJ, Lee JM, Park S, Kim ST, Kim E-J, Kim JS, Moon SH, Lee J-H, Ewers M, Na DL, Seo SW (2018) The impact of education on cortical thickness in amyloid-negative subcortical vascular dementia: cognitive reserve hypothesis. Alzheimers Res Ther 10:103. https://doi.org/10. 1186/s13195-018-0432-5

43. Kawas CH, Kim RC, Sonnen JA, Bullain SS, Trieu T, Corrada MM (2015) Multiple pathologies are common and related to dementia in the oldestold: the 90+ study. Neurology 85:535-542. https://doi.org/10.1212/WNL. 0000000000001831

44. Kliegel M, Zimprich D, Rott C (2004) Life-long intellectual activities mediate the predictive effect of early education on cognitive impairment in centenarians: a retrospective study. Aging Ment Health 8 : 430-437. https://doi.org/10.1080/13607860410001725072

45. Koepsell TD, Kurland BF, Harel O, Johnson EA, Zhou X-H, Kukull WA (2008) Education, cognitive function, and severity of neuropathology in Alzheimer disease. Neurology 70:1732-1739. https://doi.org/10.1212/01.wnl. 0000284603.85621.aa

46. Krajewska M, Smith LH, Rong J, Huang X, Hyer ML, Zeps N, lacopetta B, Linke SP, Olson AH, Reed JC, Krajewski S (2009) Image analysis algorithms for immunohistochemical assessment of cell death events and fibrosis in tissue sections. J Histochem Cytochem 57:649-663. https://doi.org/10.1369/ jhc.2009.952812

47. Kukull WA, Higdon R, Bowen JD, McCormick WC, Teri L, Schellenberg GD, van Belle G, Jolley L, Larson EB (2002) Dementia and Alzheimer disease incidence: a prospective cohort study. Arch Neurol 59:1737-1746

48. Latimer CS, Keene CD, Flanagan ME, Hemmy LS, Lim KO, White LR, Montine KS, Montine TJ (2017) Resistance to Alzheimer disease neuropathologic changes and apparent cognitive resilience in the Nun and Honolulu-Asia aging studies. J Neuropathol Exp Neurol. https://doi. org $/ 10.1093 / \mathrm{jnen} / \mathrm{n} \mid \times 030$

49. Launer LJ, Petrovitch H, Ross GW, Markesbery W, White LR (2008) AD brain pathology: vascular origins? Results from the HAAS autopsy study. Neurobiol Aging 29:1587-1590. https://doi.org/10.1016/j.neurobiolaging. 2007.03.008 
50. Li G, Larson EB, Sonnen JA, Shofer JB, Petrie EC, Schantz A, Peskind ER, Raskind MA, Breitner JCS, Montine TJ (2007) Statin therapy is associated with reduced neuropathologic changes of Alzheimer disease. Neurology 69: 878-885. https://doi.org/10.1212/01.wnl.0000277657.95487.1c

51. Liachko NF, Guthrie CR, Kraemer BC (2010) Phosphorylation promotes neurotoxicity in a Caenorhabditis elegans model of TDP-43 Proteinopathy. J Neurosci. https://doi.org/10.1523/JNEUROSCI.2911-10.2010

52. Mahoney-Sanchez L, Belaidi AA, Bush Al, Ayton S (2016) The complex role of apolipoprotein E in Alzheimer's disease: an overview and update. J Mol Neurosci 60:325-335. https://doi.org/10.1007/s12031-016-0839-z

53. Mirra SS (1997) The CERAD neuropathology protocol and consensus recommendations for the postmortem diagnosis of Alzheimer's disease: a commentary. Neurobiol Aging 18:S91-S94

54. Mirra SS, Heyman A, McKeel D, Sumi SM, Crain BJ, Brownlee LM, Vogel FS, Hughes JP, Belle G v, Berg L (1991) The consortium to establish a registry for Alzheimer's disease (CERAD): part II. Standardization of the neuropathologic assessment of Alzheimer's disease. Neurology. https://doi. org/10.1212/WNL.41.4.479

55. Montine TJ, Phelps CH, Beach TG, Bigio EH, Cairns NJ, Dickson DW, Duyckaerts C, Frosch MP, Masliah E, Mirra SS, Nelson PT, Schneider JA, Thal DR, Trojanowski JQ, Vinters HV, Hyman BT (2012) National institute on aging-Alzheimer's association guidelines for the neuropathologic assessment of Alzheimer's disease: a practical approach. Acta Neuropathol. https://doi.org/10.1007/s00401-011-0910-3

56. Montine TJ, Sonnen JA, Montine KS, Crane PK, Larson EB (2012) Adult changes in thought study: dementia is an individually varying convergent syndrome with prevalent clinically silent diseases that may be modified by some commonly used therapeutics. Curr Alzheimer Res 9:718-723

57. Munoz DG, Ganapathy GR, Eliasziw M, Hachinski V (2000) Educational attainment and socioeconomic status of patients with autopsy-confirmed Alzheimer disease. Arch Neurol 57:85-89

58. Murray ME, Graff-Radford NR, Ross OA, Petersen RC, Duara R, Dickson DW (2011) Neuropathologically defined subtypes of Alzheimer's disease with distinct clinical characteristics: a retrospective study. Lancet Neurol 10:785796. https://doi.org/10.1016/S1474-4422(11)70156-9

59. Nag S, Yu L, Boyle PA, Leurgans SE, Bennett DA, Schneider JA (2018) TDP-43 pathology in anterior temporal pole cortex in aging and Alzheimer's disease. Acta Neuropathol Commun 6:33. https://doi.org/10.1186/s40478018-0531-3

60. Nag S, Yu L, Capuano AW, Wilson RS, Leurgans SE, Bennett DA, Schneider JA (2015) Hippocampal sclerosis and TDP-43 pathology in aging and Alzheimer disease. Ann Neurol 77:942-952. https://doi.org/10.1002/ana. 24388

61. Nagy Z, Esiri MM, Jobst KA, Morris JH, King EMF, McDonald B, Litchfield S, Smith A, Barnetson L, Smith AD (1995) Relative roles of plaques and tangles in the dementia of Alzheimer's disease: correlations using three sets of neuropathological criteria. Dement Geriatr Cogn Disord. https://doi.org/10. $1159 / 000106918$

62. Negash S, Wilson RS, Leurgans SE, Wolk DA, Schneider JA, Buchman AS Bennett DA, Arnold SE (2013) Resilient brain aging: characterization of discordance between Alzheimer's disease pathology and cognition. Curr Alzheimer Res 10:844-851

63. Nelson PT, Abner EL, Schmitt FA, Kryscio RJ, Jicha GA, Smith CD, Davis DG, Poduska JW, Patel E, Mendiondo MS, Markesbery WR (2010) Modeling the association between 43 different clinical and pathological variables and the severity of cognitive impairment in a large autopsy cohort of elderly persons. Brain Pathol 20:66-79. https://doi.org/10.1111/ j.1750-3639.2008.00244.x

64. Nelson PT, Alafuzoff I, Bigio EH, Bouras C, Braak H, Cairns NJ, Castellani RJ, Crain BJ, Davies P, Del Tredici K, Duyckaerts C, Frosch MP, Haroutunian V, Hof PR, Hulette CM, Hyman BT, Iwatsubo T, Jellinger KA, Jicha GA, Kovari E, Kukull WA, Leverenz JB, Love S, Mackenzie IR, Mann DM, Masliah E, McKee AC, Montine TJ, Morris JC, Schneider JA, Sonnen JA, Thal DR, Trojanowski JQ, Troncoso JC, Wisniewski T, Woltjer RL, Beach TG (2012) Correlation of Alzheimer disease neuropathologic changes with cognitive status: a review of the literature. J Neuropathol Exp Neurol 71:362-381. https://doi.org/10. 1097/NEN.0b013e31825018f7

65. Nelson PT, Braak H, Markesbery WR (2009) Neuropathology and cognitive impairment in Alzheimer disease: a complex but coherent relationship. J Neuropathol Exp Neurol 68:1-14. https://doi.org/10.1097/NEN. Ob013e3181919a48
66. Nelson PT, Dickson DW, Trojanowski JQ, Jack CR, Boyle PA, Arfanakis K, Rademakers R, Alafuzoff I, Attems J, Brayne C, Coyle-Gilchrist ITS, Chui HC, Fardo DW, Flanagan ME, Halliday G, Hokkanen SRK, Hunter S, Jicha GA, Katsumata Y, Kawas CH, Keene CD, Kovacs GG, Kukull WA, Levey Al, Makkinejad N, Montine TJ, Murayama S, Murray ME, Nag S, Rissman RA, Seeley WW, Sperling RA, White lii CL, Yu L, Schneider JA (2019) Limbicpredominant age-related TDP-43 encephalopathy (LATE): consensus working group report. Brain. https://doi.org/10.1093/brain/awz099

67. Nelson PT, Jicha GA, Schmitt FA, Liu H, Davis DG, Mendiondo MS, Abner EL, Markesbery WR (2007) Clinicopathologic correlations in a large Alzheimer disease center autopsy cohort: neuritic plaques and neurofibrillary tangles "do count" when staging disease severity. J Neuropathol Exp Neurol 66: 1136-1146. https://doi.org/10.1097/nen.0b013e31815c5efb

68. Nelson PT, Smith CD, Abner EA, Schmitt FA, Scheff SW, Davis GJ, Keller JN, Jicha GA, Davis D, Wang-Xia W, Hartman A, Katz DG, Markesbery WR (2009) Human cerebral neuropathology of type 2 diabetes mellitus. Biochim Biophys Acta 1792:454-469. https://doi.org/10.1016/j.bbadis.2008.08.005

69. Nelson PT, Trojanowski JQ, Abner EL, Al-Janabi OM, Jicha GA, Schmitt FA, Smith CD, Fardo DW, Wang WX, Kryscio RJ, Neltner JH, Kukull WA, Cykowski MD, Van Eldik LJ, Ighodaro ET (2016) "New old pathologies": ad, part, and cerebral age-related TDP-43 with sclerosis (CARTS). J Neuropathol Exp Neurol 75(6):482-98. https://doi.org/10.1093/jnen/nlw033

70. Neltner JH, Abner EL, Baker S, Schmitt FA, Kryscio RJ, Jicha GA, Smith CD, Hammack E, KGBRull WA, Brenowitz WD, Van Eldik LJ, Nelson PT (2014) Arteriolosclerosis that affects multiple brain regions is linked to hippocampal sclerosis of ageing. Brain. https://doi.org/10.1093/brain/awt318

71. Neltner JH, Abner EL, Jicha GA, Schmitt FA, Patel E, Poon LW, Marla G, Green RC, Davey A, Johnson MA, Jazwinski SM, Kim S, Davis D, Woodard JL, Kryscio RJ, Van Eldik LJ, Nelson PT (2016) Brain pathologies in extreme old age. Neurobiol Aging. https://doi.org/10.1016/j.neurobiolaging.2015.10.009

72. Parisi JM, Rebok GW, Xue Q-L, Fried LP, Seeman TE, Tanner EK, Gruenewald $T L$, Frick KD, Carlson MC (2012) The role of education and intellectual activity on cognition. J Aging Res 2012:416132. https://doi.org/10.1155/ 2012/416132

73. Polvikoski T, Sulkava R, Haltia M, Kainulainen K, Vuorio A, Verkkoniemi A, Niinisto L, Halonen P, Kontula K (1995) Apolipoprotein E, dementia, and cortical deposition of beta-amyloid protein. N Engl J Med 333:1242-1247. https://doi.org/10.1056/NEJM199511093331902

74. Qiu W-Y, Yang Q, Zhang W, Wang N, Zhang D, Huang Y, Ma C (2018) The correlations between postmortem brain pathologies and cognitive dysfunction in aging and Alzheimer's disease. Curr Alzheimer Res 15:462473. https://doi.org/10.2174/1567205014666171106150915

75. Riley KP, Snowdon DA, Desrosiers MF, Markesbery WR (2005) Early life linguistic ability, late life cognitive function, and neuropathology: findings from the Nun study. Neurobiol Aging 26:341-347. https://doi.org/10.1016/j. neurobiolaging.2004.06.019

76. Rizzardi AE, Johnson AT, Vogel Rl, Pambuccian SE, Henriksen J, Skubitz AP, Metzger GJ, Schmechel SC (2012) Quantitative comparison of immunohistochemical staining measured by digital image analysis versus pathologist visual scoring. Diagn Pathol 7:42. https://doi.org/10.1186/1746-1596-7-42

77. Rizzardi AE, Zhang X, Vogel Rl, Kolb S, Geybels MS, Leung Y-K, Henriksen JC, Ho S-M, Kwak J, Stanford JL, Schmechel SC (2016) Quantitative comparison and reproducibility of pathologist scoring and digital image analysis of estrogen receptor beta2 immunohistochemistry in prostate cancer. Diagn Pathol 11:63. https://doi.org/10.1186/s13000-016-0511-5

78. Robinson JL, Corrada MM, Kovacs GG, Dominique M, Caswell C, Xie SX, Lee VMY, Kawas CH, Trojanowski JQ (2018) Non-Alzheimer's contributions to dementia and cognitive resilience in the 90+ study. Acta Neuropathol. https://doi.org/10.1007/s00401-018-1872-5

79. Robinson JL, Geser F, Corrada MM, Berlau DJ, Arnold SE, Lee VM-Y, Kawas CH, Trojanowski JQ (2011) Neocortical and hippocampal amyloid-beta and tau measures associate with dementia in the oldest-old. Brain 134:37083715. https://doi.org/10.1093/brain/awr308

80. Robinson JL, Molina-Porcel L, Corrada MM, Raible K, Lee EB, Lee VM-Y, Kawas CH, Trojanowski JQ (2014) Perforant path synaptic loss correlates with cognitive impairment and Alzheimer's disease in the oldest-old. Brain 137:2578-2587. https://doi.org/10.1093/brain/awu190

81. Sahoo A, Bejanin A, Murray ME, Tosakulwong N, Weigand SD, Serie AM, Senjem ML, Machulda MM, Parisi JE, Boeve BF, Knopman DS, Petersen RC, Dickson DW, Whitwell JL, Josephs KA (2018) TDP-43 and Alzheimer's disease pathologic subtype in non-amnestic Alzheimer's disease 
dementia. J Alzheimers Dis 64:1227-1233. https://doi.org/10.3233/JAD180169

82. SantaCruz KS, Sonnen JA, Pezhouh MK, Desrosiers MF, Nelson PT, Tyas SL (2011) Alzheimer disease pathology in subjects without dementia in 2 studies of aging: the Nun study and the adult changes in thought study. J Neuropathol Exp Neurol 70:832-840. https://doi.org/10.1097/NEN. 0b013e31822e8ae9

83. Schmahmann JD (2019) The cerebellum and cognition. Neurosci Lett 688: 62-75. https://doi.org/10.1016/..neulet.2018.07.005

84. Schmechel DE, Saunders AM, Strittmatter WJ, Crain BJ, Hulette CM, Joo SH, Pericak-Vance MA, Goldgaber D, Roses AD (1993) Increased amyloid betapeptide deposition in cerebral cortex as a consequence of apolipoprotein $E$ genotype in late-onset Alzheimer disease. Proc Natl Acad Sci U S A 90: 9649-9653

85. Schneider JA, Arvanitakis Z, Bang W, Bennett DA (2007) Mixed brain pathologies account for most dementia cases in community-dwelling older persons. Neurology 69:2197-2204. https://doi.org/10.1212/01.wnl. 0000271090.28148.24

86. Schneider JA, Wilson RS, Bienias JL, Evans DA, Bennett DA (2004) Cerebral infarctions and the likelihood of dementia from Alzheimer disease pathology. Neurology 62:1148-1155

87. Serrano-Pozo A, Qian J, Muzikansky A, Monsell SE, Montine TJ, Frosch MP, Betensky RA, Hyman BT (2016) Thal amyloid stages do not significantly impact the correlation between neuropathological change and cognition in the Alzheimer disease continuum. J Neuropathol Exp Neurol. https://doi. org/10.1093/jnen/nlw026

88. Smith VD, Bachstetter AD, Ighodaro E, Roberts K, Abner EL, Fardo DW, Nelson PT (2018) Overlapping but distinct TDP-43 and tau pathologic patterns in aged hippocampi. Brain Pathol 28:264-273. https://doi.org/10. 1111/bpa.12505

89. Snowdon DA, Kemper SJ, Mortimer JA, Greiner LH, Wekstein DR, Markesbery WR (1996) Linguistic ability in early life and cognitive function and Alzheimer's disease in late life. Findings from the Nun study. JAMA 275:528-532

90. Sonnen JA, Larson EB, Crane PK, Haneuse S, Li G, Schellenberg GD, Craft S, Leverenz JB, Montine TJ (2007) Pathological correlates of dementia in a longitudinal, population-based sample of aging. Ann Neurol 62:406-413. https://doi.org/10.1002/ana.21208

91. Sonnen JA, Larson EB, Haneuse S, Woltjer R, Li G, Crane PK, Craft S, Montine TJ (2009) Neuropathology in the adult changes in thought study: a review. J Alzheimers Dis 8(3):703-11. https://doi.org/10.3233/JAD-2009-1180

92. Sonnen JA, Santa Cruz K, Hemmy LS, Woltjer R, Leverenz JB, Montine KS, Jack CR, Kaye J, Lim K, Larson EB, White L, Montine TJ (2011) Ecology of the aging human brain. Arch Neurol 68:1049-1056. https://doi.org/10.1001/ archneurol.2011.157

93. Taylor LM, McMillan PJ, Liachko NF, Strovas TJ, Ghetti B, Bird TD, Dirk Keene C, Kraemer BC (2018) Pathological phosphorylation of tau and TDP-43 by TTBK1 and TTBK2 drives neurodegeneration. Mol Neurodegener. https://doi. org/10.1186/s13024-018-0237-9

94. Teng EL, Hasegawa K, Homma A, Imai Y, Larson E, Graves A, Sugimoto K, Yamaguchi T, Sasaki H, Chiu D (1994) The cognitive abilities screening instrument (CASI): a practical test for cross-cultural epidemiological studies of dementia. Int psychogeriatrics 6:45-58 discussion 62

95. Thal DR, Rüb U, Orantes M, Braak H (2002) Phases of Aß-deposition in the human brain and its relevance for the development of AD. Neurology. https://doi.org/10.1212/WNL.58.12.1791

96. Uchino A, Takao M, Hatsuta H, Sumikura H, Nakano Y, Nogami A, Saito Y, Arai T, Nishiyama K, Murayama S (2015) Incidence and extent of TDP-43 accumulation in aging human brain. Acta Neuropathol Commun 3:35. https://doi.org/10.1186/s40478-015-0215-1

97. Wennberg AM, Tosakulwong N, Lesnick TG, Murray ME, Whitwell JL, Liesinger AM, Petrucelli L, Boeve BF, Parisi JE, Knopman DS, Petersen RC, Dickson DW, Josephs KA (2018) Association of Apolipoprotein E epsilon4 with Transactive response DNA-binding protein 43. JAMA Neurol 75:13471354. https://doi.org/10.1001/jamaneurol.2018.3139

98. White L, Petrovitch H, Hardman J, Nelson J, Davis DG, Ross GW, Masaki K, Launer L, Markesbery WR (2002) Cerebrovascular pathology and dementia in autopsied Honolulu-Asia aging study participants. Ann N Y Acad Sci 977:9-23

99. White LR, Edland SD, Hemmy LS, Montine KS, Zarow C, Sonnen JA, UyeharaLock JH, Gelber RP, Ross GW, Petrovitch H, Masaki KH, Lim KO, Launer LJ, Montine TJ (2016) Neuropathologic comorbidity and cognitive impairment in the Nun and Honolulu-Asia aging studies. Neurology 86:1000-1008 https://doi.org/10.1212/WNL.0000000000002480

100. Wilson RS, Yu L, Lamar M, Schneider JA, Boyle PA, Bennett DA (2019) Education and cognitive reserve in old age. Neurology. https://doi.org/10. 1212/WNL.0000000000007036

101. Wilson RS, Yu L, Trojanowski JQ, Chen E-Y, Boyle PA, Bennett DA, Schneider JA (2013) TDP-43 pathology, cognitive decline, and dementia in old age. JAMA Neurol 70:1418-1424. https://doi.org/10.1001/jamaneurol.2013.3961

102. Yang H-S, Yu L, White CC, Chibnik LB, Chhatwal JP, Sperling RA, Bennett DA, Schneider JA, De Jager PL (2018) Evaluation of TDP-43 proteinopathy and hippocampal sclerosis in relation to APOE epsilon4 haplotype status: a community-based cohort study. Lancet Neurol 17:773-781. https://doi.org/ 10.1016/S1474-4422(18)30251-5

103. Yu L, De Jager PL, Yang J, Trojanowski JQ, Bennett DA, Schneider JA (2015) The TMEM106B locus and TDP-43 pathology in older persons without FTLD. Neurology 84:927-934. https://doi.org/10.1212/WNL.0000000000001313

\section{Publisher's Note}

Springer Nature remains neutral with regard to jurisdictional claims in published maps and institutional affiliations.

\section{Ready to submit your research? Choose BMC and benefit from:}

- fast, convenient online submission

- thorough peer review by experienced researchers in your field

- rapid publication on acceptance

- support for research data, including large and complex data types

- gold Open Access which fosters wider collaboration and increased citations

- maximum visibility for your research: over $100 \mathrm{M}$ website views per year

At BMC, research is always in progress.

Learn more biomedcentral.com/submissions 